\title{
LETTERATURA DEGLI ESULI ITALIANI A MALTA DURANTE IL RISORGIMENTO
}

\author{
Oliver Friggieri
}

Sotto la dominazione dell'Inghilterra, l'isola non solo rimase sempre legata alla Sicilia per vincoli di lingua e di costumi, ma partecipò alla rigenerazione della penisola. ${ }^{1} \mathrm{Il}$ paese, nonostante ciò, pur avendo una lunga tradizione italiana affidata ininterrottamente alla classe borghese, non possedeva in sé le qualità di direzione e di coraggio che avrebbero potuto avviarlo a formare una coscienza nazionale capace di realizzare una trasformazione politica e culturale. Il basso livello dell'istruzione delle masse. ${ }^{2}$ il dislivello sociale tra la classe colta e la classe dei lavoratori, nonché l'incuranza in cui si trovava da secoli l'idioma maltese come strumento di unificazione e di civilizzazione, sono alcune delle cause della rassegnazione e della indolenza quasi naturale che caratterizzavano il popolo. Quando, poi, questa insularità tradizionale cominciava ad essere rotta, ebbe inizio una profonda riforma in sede politica e culturale. Mentre continuava a svolgersi la cultura italiana locale, si cominciavano a seminare i primi germi per una nuova cultura locale, scritta in maltese, benchè identificabile mediante le caratteristiche dell'antica cultura che era considerata da molto tempo come l'unica dell'isola. Il movimento a favore del maltese diede inizio anche ad una presa di coscienza patriottica a proposito della situazione costituzionale.

Il parallelismo tra cultura italiana in Italia e la sua modificazione a Malta si svolgeva su due piani: continuazione e adattamento nuovo a livello letterario, e imitazione e conseguimento a livello politico. A causa del rapporto che il secolo XIX stabiliva tra espressione letteraria e attività patriottica, il principio fondamentale del nazionalismo romantico si identificò del tutto con la visione di una letteratura d'impegno comunitario. Scrivere cominciava a significare anche combattere, politicizzare la parola.

1. Cfr. E. Librino, Malta nel risorgimento italiano dai carteggi dell'Archivio di Stato di Palermo, “A.S.M.” X, fasc. III-IV, p. 257.

2. Cfr. I rapporti dei commissionari inglesi, particolarmente P.G. Julyan, Report on the civil establishments of Malta, London, W. Clowes \& Sons, 1879; P.J. Keenan, Report upon the educational system of Malta, Dublin, Alexander Thom, 1879. 


\section{Cominciano ad arrivarse gli esuli}

Sono diversi i motivi che costringevano gli esuli liberali italiani a stabilirsi a Malta durante il periodo $1804-1860$ e poco dopo, fra i quali il fatto che l'arcipelago maltese offriva un sicuro rifugio sotto il dominio dell'Inghilterra liberale; da Malta potevano osservare da vicino lo svolgimento delle vicende, ricevere comunicazioni e notizie, e mantenere un vivo contatto con le famiglie e gli amici in patria, essendo anche in grado di ritornare di tempo in tempo. ${ }^{3}$ C'è anche l'identità di cultura, di lingua e di costumi, come attestano diversi documenti della Chiesa e del governo britannico a Malta, ad esempio una lettera dell'arcivescovo Pubblio Maria de' Conti Sant al Governatore O'Ferrall (1848) e un dispaccio che il Sant inviò al ministro inglese delle Colonie Grey il 29 novembre $1849 .^{4}$

L'emigrazione politica del Risorgimento ebbe inizio durante il periodo napoleonico. Si ritiene che il primo esule sia stato Vittorio Barzoni che, dopo essere stato sfrattato đa Vienna da Napoleone, ${ }^{5}$ arrivò a Malta nel 1804 e vi svolse una vasta attività giornalistica per circa dieci anni. Era uno degli emigrati anti-francesi e a favore degli inglesi, ma aveva anche le sue idee liberali a proposito dell'insurrezione italiana, di cui diede prova nei suoi scritti pubblicati sul "Giomale di Malta" (1813). Il Barzoni iniziò anche il giornale "L'argo" e lo utilizzò nella propaganda contro la supremazia napoleonica. Tra îl 1804-1810 pubblicò "I1 cartaginese - giornale politico" in cui, fra altre cose, predicava la necessità di una Italia unita. Nel 1813 iniziò la pubblicazione di un foglio governativo, la "Gazzetta del Governo di Malta" che poi nel 1816 assumeva un nome inglese, "Malta Government Gazette". Durante il suo soggiorno nell'isola Barzoni scrisse due libri, Operette (1808) e Dissertazione politica (1811).

Altri ribelli si rifugiavano a Malta nel 1815, a causa della Restaurazione. Il primo gruppo di rilievo, importante per l'effetto che lasciò, è quello che vi giunse dopo le insurrezioni piemontesi del 1820-1821. Dopo il crollo del regime napoleonico in Europa, e gli insuccessi delle rivolte dei Carbonari a Napoli nel 1820, in Piemonte nel 1821 , e negli stati papali nel 1830 , iniziò un lungo periodo di govemo poliziesco durato quasi fino al 1860 . Durante questo periodo avvenne il primo grande esodo

3. G. Patti, I cento giorni di Garibaldi in Sicilia nel giornalismo maltese, Messina, "La Sicilia" 1972, p. 14

4. I due documenti sono riprodotti da G. Mangion, Governo inglese, risorgimento italiano ed opinione pubblica a Malta 1848-1851, Malta, Casa S. Giuseppe, 1970, rispettivaemente pp. 50-51 e 60-61.

5. B. Cellini, recensione di L. Giuliano, Il comitato mazziniano di Malta, "La Sicilia nel risorgimento italiano", gennaio-giugno 1932, "A.S.M." VI, fasc I, 1935, p. 61. 
verso Malta. Erano scrittori e professionisti, o rivoluzionari attivi. Fra i nomi più importanti ci sono Michele Carascosa, Raffaele Poerio, Gabriele Rossetti e altri. Il Rossetti fuggì a Malta il 20 aprile 1821. Fu accolto entusiasticamente dal pubblico, particolarmente negli ambienti letterari. Fin dal principio apparve chiaro che la sua presenza nell'isola si appoggiava alla fama poetica. Si dice che il suo arrivo nel porto creò una folla commossa e plaudente e ad un tratto, "da una agil feluca, gremita di donne leggiadre, sentii sciogliersi (...) un suo canto già famoso:

Sei pur bella cogli astri sul crine che scintillan quai vivi zaffiri, è pur dolce quel fiato che spiri porporiera foriera del dì. ${ }^{6}$

A Malta fu protetto dalle autorità britanniche, e vi dimorò per oltre due anni durante i quali avvicinò studiosi maltesi, fra cui il poeta Luigi Rigord, e tenne diverse accademie poetiche, componendo, fra l'altro, L'amore che dipinge la bellezza e parecchi epigrammi. Sir Hookham Frere, uno studioso inglese residente nell' isola (a cui il poeta dedicò il suo Tempo, ovvero Iddio e l'uomo), diventò suo protettore presso gli inglesi. La sera del 12 agosto 1821, nella casa del Cavaliere Parisio, "gremita di dame, letterati e diplomatici", ìl "decano dell'emigrazione italiana" cantò l'ode L'apostolo san Paolo che naufraga a Malta e se ne dichiara il protettore.?

Intorno alla sua attività poetica nell' ambiente dei letterati maltesi e degli esuli, il Rossetti scrisse così in una lettera del 28 marzo 1822 a Giacomo Ferretti: "Accademie di poesia estemporanea e scuole di lingua e letteratura sono state il mio ricovero qui; e colà vì aggiungerò stampa di mie opere non poche, e così faremo schermo contro i colpi del Fato. Di molti canti che ho improvvisato, e che hanno trascritto, le cure dell'amicizia ospitale han dato (me inscio) alla stampa il canto che a te spedisco". 8

6. A. Sautto, Gabriele Rossetti nell'isola di Malta, "M.L.", II serei, a. V, n. II, 1930, p. • 322.Per il brano citato, cfr. La costituuzzione in Napoli e esilio in Malta, vv. 223-230.

7. B. Fiorentini, Malta rifugio di esuli e focolare ardente di cospirazione durante il risorgimento italiano, Malta, Casa S. Giuseppe 1966, pp. 32-33; e L. Sciavone, Esuli italiani a Malta durante il risorgimento, Malta, Società Dante Alighieri, 1963, p.159. Il nome della poesia ricorda l'occasione: L'apostolo San Paolo che naufraga in Malta e se ne dichiara il protettore, canto estemporaneo di Gabriele Rossetti - ad argomento dato, tratto a sorte da un'urna, ove molti ve n'erano, nella sera del 12 agosto del J82J. in casa del Cavalier Parisio, innanzi a scelta adunanza, Malta, 1822.

8. Citata da A. Sautto, op. cit., p. 323. 
Raffaele Poerio venne a Malta varie volte durante i primi sette anni del suo lungo esilio. La prima volta fu verso il 20 gennaio 1822 quando, dopo essersi ritirato sui monti della Calabria, poté sfuggire alle ricerche della polizia borbonica. Nel maggio 1822 tornava nell'isola, ma anche questa volta non vi si poteva fermare a lungo. Nell'aprile 1823 , incaricato di una missione da parte dei compagni d'esilio, approdava ancora a Malta, ma le insistenze del console napoletano affinché il Poerio lasciasse l'isola ottennero finalmente il loro risultato, e il 21 aprile il calabrese partiva per la terza volta. Il Rossetti e il De Luca lo prendevano sotto braccio, e tutti gli altri andavano appresso. Approdò di nuovo a Malta nel maggio 1823; e nel luglio venne arrestato, condotto in prigione e tenuto in custodia. Nonostante ciò, tornava ancora negli anni successivi e vi si tratteneva più lungamente. ${ }^{9}$

Il primo esule irpino fu D. Pietro De Luca di Montefusco, arrivato il 24 febbraio 1823. Fra gli amici con i quali s'incontrava, trovò il Rossetti, occupato nell'insegnamento della lingua e della letteratura italiane. Il 29 ottobre 1924 se ne andò verso il Levante. ${ }^{10}$ Il Rossetti dedica tutta la settima parte (strofe 210-243) del poema La vita mia ad una elaborata narrazione delle sue esperienze nell'isola, così descritta nella sestina che introduce l'intero canto:
A te si volse in pria l'anglica prora, florida Malta, piccola ma bella;
fra l'inquieto mar questa dimora, d'italo genio e d'araba favella.
Fra le menzogne meditando il vero, in te trascorsi un mezzo lustro intero. ${ }^{11}$

Questa non fu l'unica volta che il Rossetti prese uno spunto da avvenimenti politici e sociali maltesi; in occasione delle nozze di due personaggi inglesi, membri dell'alta classe, compose una lunga ode che, nel suo allontanare il fatto dalle condizioni reali, nell'atmosfera mitica riallacciata al gusto neoclassico, e nell'onda melodiosa dei

9. E. Michel, Raffaele Poerio esule a Malta 1822-1823, “A.S.M.”, VII, fasc. I, 1929, pp. 47-61; e VIII, fasc. III, 1930, pp. 215-225.

10. V. Cannaviello, I rivoluzionari irpini del 1820 esuli a Malta "A.S.M.", II, fasc II-III, 1931, pp. 102-107

11. Tutto quanto il Rossetti rivela nella settima parte del poema, conferma cio che scrive il figlio Guglielmo Michele: "Era tanto noto come improvvisatore quanto come poeta (egli continuo" a improvvisare per qualche tempo anche dopo la sua venuta a Londra) e questo, con gli altri doni, lo rese popolare nella societa maltese" (Gabriele Rossetti e $i$ suoi parenti. Opere inedite e rare di Gabriele Rossetti - La vita mia - Il testamento, a c. di D.Ciampoli, Lanciano, Carabba, 1910. p. 169). 
settenari piani e sdruccioli, ricorda le due odi A Luigia Pallavicini caduta da cavallo e All'amica risanata che il Foscolo aveva pubblicato circa venti anni prima. Da una circostanza particolare che mostra che il poeta non si ritenne distaccato dallo svolgersi della vita sociale dell'̀̀sola, il Rossetti creò una vìsione universale:

\author{
Scendi, e l'aurato talamo \\ del lume tuo rischiara; \\ ve' che le Grazie a gara \\ t'infioran il sentier, \\ Pubero Dio tedifero \\ cui brilla il gaudio in viso; \\ il cui divin sorriso \\ santifica il piacer ${ }^{12}$
}

Il generale Michele Carascosa, che già aveva seguìto Napoleone a Malta, vi si rifugiò dopo i moti del 1821 e sposò la figlia del generale maltese Gatt. ${ }^{13}$ Salvatore Chiudemi, scrittore, traduttore e storico, riparò a Malta, donde passò a Torino e insegnò storia nell'università. Guglielmo Finotti, ferrarese, dopo quattro anni di soggiorno in Tunisia, venne a Malta, fondò due fogli, "Il corriere mercantile di Malta" e "L'educatore", pieni di spirito patriottico, e stampò alcuni libri, fra i quali Reggenza di Tunisi. ${ }^{14}$

Nel gruppo di messinesi che giunsero a Malta nel 1822 c'era Giuseppe Cesareo, il padre del poeta maltese Paolo, nato a Floriana nel 1844. Paolo conseguì i suoi studi a Siracusa sotto la direzione del poeta Emanuele Giaracà; e fu amico degli scrittori Sebastiano Macaluso e Emilio M. Di Natale, che ha pubblicato parecchi scritti letterari su riviste maltesi. ${ }^{15}$

Insieme all'attività politica degli esuli nell'isola, va anche la loro partecipazione letteraria. Molti di loro fondarono scuole private, e tennero diverse accademie di

12. G. Rossetti, Per le faustissime nozze del Right Honourable J. Lord Wallscourt e Miss Lockode epitalamica, Malta, 1822, p. 1.

13. A Malta probabilmente scrisse Memoires historiques politiques et militaires sur la revolution du Royaume de Naples en 1820 et 1821, che pubblico`a Londra nel 1823 (cfr. L. Schiavone, op. cit., p. 128).

14. E. Rossi, Lingua Italiana, dialetto maltese e politica britannica a Malta, Livorno, Giusti, 1929, p.57. Il Finotti si trovò nuovamente a Malta nel 1874 e diede alle stampe diversi opuscoli e tenne varie conferenze pubbliche.

15. R. Mifsud Bonnici, Dizzjunarju bijo-bibljografiku nazzjonali, Malta, Dipartiment ta' 1-Informazzjoni, 1960, p. 127. 
poesia e di critica dantesca. Gli scrittori si dedicarono alla stesura di opere creative, che spesso servirono a diffondere la cultura generale e a instillare fra i maltesi il sentimento della nazionalità. Ad opera dei primi profughi fiorirono le accademie di poesia estemporanea, tradizionalmente cara al pubblico locale che si divertiva a suggerire un tema, normalmente attinente a importanti eventi politici e religiosi, in base ai quali il poeta era invitato a comporre versi. Il poeta esprimeva quasi sempre le pene dell'esilio e la speranza in un avvenire glorioso e libero. Tali composizioni diventavano poi oggetto di vivaci commenti. Molti giovani si appassionarono a raccoglierle, e alcune di esse furono pubblicate come fogli e libretti. ${ }^{16}$ Uno di questi poeti fu Giovanni Giustiniani, da Imola. In L'esule, cerca con fervore di suscitare un senso di ammirazione per i cittadini che vanno in fuga di paese in paese, rievocando la speranza in un futuro vittorioso:

Non vedete la livide nube sovra il capo de' vostri nemici

non udite de' Bardi le tube

fra le libere insegne vittrici?

Chi non spera la gloria primiera, non è degno dell' italo onor. ${ }^{17}$

Un altro poeta italiano che svolgeva una simile attività presso i circoli culturali maltesi, fu Camillo Mapei. Era noto fra gli arcadi con il nome di Narizio Ismeneo. Tenuto d'occhio dai Borboni, essendo sospettato di nutrire simpatie per la "Giovine Italia", il Mapei aggravò la sua posizione tenendo una conferenza, piuttosto aggressiva, sulle glorie antiche dell'Italia. Con l'aiuto di alcuni amici che avevano dimora a Marsiglia, giunse a Malta alla fine del maggio 1841; nell'isola, a causa della sua abilità di poeta estemporaneo, era ben visto dalle autorità inglesi e dal clero locale. ${ }^{18}$ Una sua raccolta poetica mette in evidenza il fatto che era uno dei poeti più acclamati nelle accademie tenute in quegli anni. Nell'introduzione al libro Versi estemporanei, gli editori maltesi dicono così di lui: «Camillo Mapei, nome conosciuto nelle più celebri accademie d'Italia, la sera del 26 giugno improvvisava in questo Real Teatro di Malta esercendo la prima volta per necessità quella professione che solo per diletto aver altrove esercitato. Acceso di fantasia, fecondo di mente, caldo di affetti dettava nel bollor della ispirazione carmini ardenti. Scevro d'impostura, che pur suol essere il comune retaggio degli improvvisatori, mostrava spontaneità di metro, semplicità di

16. B. Fiorentini, op. cit., p. 32-33

17. Poesie estemporanee del dottor Giustiniani da Imola, cantante nella sala del club la sera del 22 febbraio in Malta, Malta Samperia del Governo, 1836, p. 7.

18. B. Fiorentini, op. cit., p. 102. 
stile, purità di lingua, per cui meritò gli applausi dei più saggi. Noi, si come è nostro costume, raccogliamo i colui versi. ${ }^{19}$ Accanto ad argomenti vari, il Mapei trattò anche l'argomento politico. La Sicilia nel 1837 è una canzonetta che si svolge in chiave musicale, esortando gli ascoltatori a rispondere generosamente alla chiamata dell'Italia e ad accettare la vocazione di costruttori della patria:

Udite, udite o secoli

I'Italia a schiava ancora, perche intestina e indomita

la fiamma la divora, ma se congiunti insorgono tutti cadranno i re.

Dopo ogni rivolta che avvampava in Italia, fino alla realizzazione dell'unità, Malta riceveva afflussi di nuovi esuli. Falliti i moti dell'Emilia e della Romagna nel 1831, arrivo` il secondo gruppo importante di profughi, questa volta da regioni lontane. E` significativo l'arrivo di tre di loro: Emilio Usiglio, Nicola Fabrizi e Tommaso Zauli Sajani, tutti responsabili del fatto che Malta presto divenne uno dei centri emigratori più noti, e che l'emigrazione politica italiana cominciò a seguire da vicino le idee $\mathrm{e}$ i metodi del Mazzini. ${ }^{20}$ Nello stesso anno il Mazzini fondò la "Giovine Italia", dopo essere stato arrestato e imprigionato per cospirazione dal Governo piemontese e poi esiliato. Il Mazzini cominciò a propagare il suo credo di unità, libertà e indipendenza e promise la sua lealtà a Carlo Alberto che in quell'anno saliva al trono del Piemonte. Ma Carlo Alberto non era in grado di aderire al programma, e il Mazzini fu costretto a fondare la "Giovine Italia", un' associazione molto diffusa che intendeva sviluppare l'attività dei Carbonari facendosi raggiungere dal maggior numero possibile di persone, traducendo tutta la causa in un ideale quasi religioso. ${ }^{21}$ Emilio Usiglio, di Modena, giunse a Malta nel 1836, quando l'isola era già nota come centro di propaganda mazziniana, come risulta da una lettera del Mazzini del 1 gennaio 1837 all'amico Melegari. Usiglio lasciò il paese nel 1842, e la sua missione fu continuata da Nicola Fabrizi, da Modena, che nel 1837 aveva progettato una spedizione armata per 1'Italia. Il Fabrizi, membro attivissimo della "Giovine Italia" che abitava al n. 183 di strada san Paolo (Valletta), una delle strade principali della capitale, si manteneva in diretto contatto con il Mazzini e con altri esuli italiani in Spagna, Montevideo, New York e

19. Versi estemporanei di Camillo Mapei, cantati nel real teatro di Malta la sera del 26 giugno, Malta, P. Cumbo, 1841 , p. 3. Un'altra sua pubblicazione che diede alle stampe a Malta, è Apologia delle lezioni sacro-morali pronunziate nella chiesa del Gesù, Malta, Stocker Bros. \&. Co., 1842.

20. G. Mangion, op. cit, pp. 4-5.

21. D. Richards, Modern Europe 1789-1945, 5a ed., London, Longmans, 1963, pp. 147-148. 
altri paesi. ${ }^{22} \mathrm{Da}$ Malta dirigeva anche vari complotti di cospirazione nella penisola. Tornato in Italia nel 1848 - 1849, riprese la via dell'esilio, fermandosi a Malta più a lungo. Nel 1860 sollecitò da Garibaldi la spedizione in Sicilia e sbarcò a Pozzallo nel giugno 1860 con venticinque compagni e mille fucili, unendosi poi a Garibaldi. Fra quelli che parteciparono a questa spedizione ci furono due maltesi, Giuseppe Camenzuli e Giorgio Balbi; il primo divenne più tardi colonnello nell'esercito italiano e morì nel terremoto messinese; e il secondo si ritirò a Malta dopo aver sposato una donna di Messina. ${ }^{23}$

\section{L'attività di Zauli Sajani}

Tommaso Zauli Sajani, da Forlì, scrittore prolifico, avendo preso parte alle insurrezioni del 1831-1832 e partecipato alle attività della "Giovine Italia", fondò a Malta "Il Mediterraneo - Gazzetta di Malta", organo della società mazziniana, e si inserì completamente nella vita culturale dell'isola (1836-1846). Lo Zauli Sajani, oltre ad essere stato un collaboratore del "Mediterraneo"(1838-1845) e autore degli articoli nella sezione 'Malta', fu anche fondatore e direttore della rivista letteraria "La speranza" (1846-1847) la cui pubblicazione finì in seguito all'amnistia concessa da Pio IX, quando potè ritornare in patria. Fra le opere che scrisse e pubblicò a Malta, ci sono: Quadri storici dell'incivilimento moderno (1846), Intorno all'attuale condizione politica dello stato della Chiesa (1846), Intorno allo stato attuale delle lettere in Italia (1846), La Valette o i Turchi a Malta nel 1565 (1850), Dizionario corografico (1840), Leggenda in quattro canti - La Grotta d'Assano (1846). ${ }^{24}$

L'opera poetica che mostra maggiormente il suo interesse a ispirarsi al vero storico più che al vero immaginato, e a integrare la propria esperienza di esule con la vita di poeta, è Il trionfo della grazia, ossia l'ultimo degli arabi in Malta (1847). L'autore dichiara d'aver preso l'argomento dalle tradizioni storiche legate a monumenti che si trovano a Malta, e da una leggenda che riguarda Ghar Hasan, una grotta in cui si dice visse un arabo dopo che i suoi compagni furono espulsi dall'isola. ${ }^{25}$ I suoi endecasillabi sciolti sono semplici, privi dell'architettura ricercata di lunghi periodi grammaticali e ritmici che appesantiscono il corso narrativo; e, pur non avendo qualità poetiche considerevoli, mantengono il tono aulico e la catena degli enjambment. Non riesce,

22. A. V. Laferla, British Malta, I, Malta Govt. Printing office 1938, p.111, sgg.

23. E. Rossi, op. cit., pp. 60-61.

24. Per queste e altre opere, cfr. G. ZAULI SAIANI, Note cronologiche su la vifa e su Ie opere di Tommaso Zauli Sajani, Forli, Tip. Democratica, 1912, pp. 17-22.

25. II trionfo della grazia, ossia ultimo degli arabi in Malta, leggenda epica di Tommaso Zauli Sajani, Malta, P. Cumbo, 1847, p. 6. 
da un punto di vista strettamente politico, a velare i suoi interessi nazionalistici, cioè l'amalgamazione di Malta con la penisola, e a guardare Malta indipendentemente dai suoi rapporti con la causa risorgimentale contemporanea:

Bianca rosa dell'italo oriente,

Melita, che ti specchi in mezzo al mare,

esce da tuo candor pallido un lume

cui dalla mia terra natal ricorda

il sorriso che muor sotto il profano

prepotente desio dello straniero

che la bacia e tormenta (...).

Un giono,

ricongiunta alla tua madre sarai,

la più misera sempre e la più bella

fra le figlie del sol.

Nonostante ciò, lo Zauli Sajani, a causa del suo coinvolgersi nella vita pubblica e culturale dell'isola, non fu sempre compreso e apprezzato da una considerevole parte della popolazione, anzi può essere considerato come un personaggio di forti contrasti che mette in evidenza le implicazioni della presenza degli esuli. Un atroce attacco contro di lui apparve nel 1842 sulla gazzetta conservatrice, rigidamente cattolica, "L'osservatore maltese", avendo l'autore presentato in un teatro la sua tragedia Lisleadamo, ${ }^{26}$ ispirata alla vita di uno dei Gran Maestri che dominavano l'isola durante l'epoca dell'Ordine Gerosolimitano: “Questo fuoruscito forestiere, è un tal Zauli Sajani da Forlì, sedicente avvocato, il quale per rendere debite grazie ai maltesi che l'hanno raccolto, molto meglio di quello si meritava; ha spalancato quivi bottega, ove va spacciando come un cerretano le sue letterarie fanfaluche cui, per nostra sventura, si è aggiunta anche la sua mogliera (...). Una parola su questa femmina autrice pseudo-romantica di quell'ammasso di scempiaggini, siccome sono Gli ultimi giorni de' Cavalieri... Ma lasciamo star da banda questo libraccio che il S. Offizio vuol mettere nel ruolo de' condannati: questo sciocco romanzo, che è un insulto il più impertinente, fatto all'onore nazionale dei maltesi ed al senso comune."27

L'attacco è tipico dell'atrocità giornalistica dell'epoca, e indica il duplice fatto che la presenza degli esuli non suscitò soltanto un vasto movimento di simpatia e

26. Lisleadamo, primo Gran Maestro dell'Ordine di S. Giovanni Gerosolomitano a Malta, Malta, Stocker Bros \& Co., 1842.

27. Critica della così detta tragedia 'II Lisleadamo' del così detto avvocato Zauli Sajani, “0. M.", 23/7/1842,p. i:.. 
di partecipazione (politiche e culturali), ma anche un contegno di controversia e di avversione dai conservatori. L'attacco contro "questo scrittabolo (...) briaco scrittore" con il negare ogni valore estetico alla tragedia: questa supposta tragedia non ha nessuna di quelle qualificazioni e le si potrebbe dare titolo siffatto: quindi non essendo nè poema ne molto meno lirica si deve confessare che è un vero pasticcio ed un vero caos indigesto (...). È antistorica e contraria a tutti i documenti che ci vengono forniti dagli annali della vita di l'Isleadamo e dalla serie di quei tempi." 28

Lo Zauli Sajami si servì della tragedia storica per esprimere due stati d'animo quello personale, cioè di patriota che sta aspettando i risultati dei moti rivoluzionari; e 1'altro, che egli intendeva diffondere fra i maltesi, cioé l'ansia di vederli insorgere contro il dominio straniero combattere per i diritti della liberta' nazionale. Per arrivare al suo ideale, adoperò una trama di emozioni proprie e la proiettò su caratteri e situazioni che avevano il loro punto di partenza nella storia, ma che si svolgevano secondo le tendenze della sua fantasia. L'opera include diversi brani che s'indirizzano alla folla e che l'invitano a organizzarsi contro le autorità locali:

\author{
Memorie atroci! \\ Che non patimmo sotto il ferreo giogo \\ di vassalli superbi, avari, crudi, \\ rotti ad ogni libidine? Venduti, \\ e rivenduti, in ludibrio fatti \\ ora di ladri, ora d'impure voglie, \\ da Pilato passando a Caifasso.
}

D'altro canto, l'impostazione dell'attacco dell" "Osservatore maltese", significativa perchè indicava quali erano $\mathrm{i}$ criteri letterari dcgli intellettuali maltesi che, a causa delle forti influenze religiose e dell'aderenza ad una visione etica definita, applicabile necessariamente ad opere creative, almeno in questo caso concludevano che un testo contenente sentimenti morali non accettabili dalla fede della maggioranza, doveva essere automaticamente considerato privo di ogni merito estetico.

28. Ibid., pp. 9-12. Per un simile attacco costruito sulla stessa concezione estetica, cioé che la verità fantastica non deve oltrepassare i limiti imposti dalla verità storica, cfr. Osservazioni al 'Lisleadamo' - tragedia del signor avv. Zauli Sajani, rappresentata nel Teatro di Malta nel 2 maggio del 1842, scritte dall'architetto ingegnere Giorgio Grongnet de Vasse, Malta, G. Camilleri, 1842. II Grongnet, fra l'altro, scrive: "II poeta che vuole abbellire e limar la sua tragedia da ogni neo [...] deve certamente adattare ad ogni personaggio quelle virtú e quei vizi che gli convengono, e di che la storia ce lo dipinge capace" (p. 6). 
L'autore forlivese reagì e aprì una causa legale contro l'editore del giornale, ma la manovra legale fallì e l'editore trovò una seconda occasione per identificare la causa del critico letterario con quella del moralista o addirittura del difensore della religione: "L'ultima prosecuzione mossaci contro dal Dott. Pantalone Bruno ad istanza del cosi' detto avvocato Zauli Sajani ha contribuito a vieppiù legar noi colla causa de' buoni maltesi, colla causa di tutti i cattolici e di ogni cittadino, il quale aborre di vedere calpestato nel fango l'onore dell'Ordine Gerosolimitano e il santo nome della Religione. Noi siamo stati (...) denunziati, trascinati dinnanzi a una corte criminale e vessati da due, Bruno e Sajani, e perchè? (...) Noi perciò siamo gettati in un mare di amarezze, dopo di aver difesa la religione dei nostri padri, e i sacramenti della nostra fede, dopo di aver svelata la sfacciataggine dello scrittore della tragedia, che ha trattato le nostre donne come sgualdrine, e i nostri preti come gl'infrattori del suggello della confessione, il venerabile anzi beato Lisleadamo come un adultero, un incestuoso, un fratricida, un tiranno; noi, sí noi siamo stati tradotti in giudizio, per aver amata la nostra patria, e la nostra religione (...). Tutto il popolo, tutto il pubblico, tutta Malta stava dal lato nostro nel giorno della causa. I più onesti, i più dotti ed integerrimi cittadini ci circondavano e quattro de' migliori avvocati del nostro foro si levarono a difendere generosamente la nostra causa, che è la causa della nazione, della religione, dell'onestà." 29

La rabbia del giornale, come fu detto, toccò anche la moglie di Tommaso, Ifigenia, anch'essa scrittrice attiva che contribuì assiduamente al movimento letterario durante il suo soggiorno a Malta con il marito, e vi pubblicò parecchi libri, fra i quali: Clelia, ossia Bologna nel 1833 (1844), II ritorno dell'emigrato (1842), Beatrice A lighieri - racconto storico del sec. XIV (1842), e il romanzo che suscitò lo sdegno dell " Osservatore maltese", Gli ultimi giorni dei cavalieri di Malta $(1840) \cdot{ }^{30}$ Beatrice Alighieri è costruito su una visione emotiva e si svolge in un'atmosfera di delicatezze, di sentimenti tenui e di azioni forti, pur non essendo privo di un linguaggio retorico e classicheggiante. Più impegnato, sia letterariamente sia politicamente, è Il ritorno dell'emigrato in cui la Zauli Sajani trasferisce la propria esperienza di esule e la colloca su un livello opposto e analogo: un personaggio maschile, che è maltese, costretto ad andare in esilio in Italia. Fedele alla tradizione dei romanzieri storici che nascondevano, pur lasciandolo trasparire, il loro intento politico nel complesso di caratteri e di situazioni extrapersonali e sorpassate, la scrittrice ritiene il parallelismo

29. Un dovere di riconoscenza, "0.M.", 14/10/1842, p. 49.

30. Il romanzo venne pubblicato a puntate su "II mediterraneo" dall'edizione del 10/10/1838, offrendo così un'altra indicazione dell'assidua collaborazione tra gli esuli e i giornalisti e i politici maltesi. 
tra la propria esperienza e quella di un giovane maltese pieno di coraggio di fronte alla sfida nazionale. II racconto puo essere definito come un'elegia dell'esilio in cui lamenti, sospiri amorosi e idealità patriottiche si intrecciano insieme per produrre una tessitura di ammirevoli gesta. Il suo duplice scopo si fa evidente fin dal primo paragrafo del racconto: "Correva il dì 6 di ottobre del 1839 - il dì che io doveva lasciar Malta mia patria per recarmi, secondo che voleva un destino fatto a me da me medesimo, alla classica terra d'Italia. Io tremava all'idea di dovermi distaccare dal luogo delle mie prime, delle mie care affezioni..." 31

Più aperto e diretto è il messaggio nazionalistico e risorgimentale del romanzo in cui le implicazioni che deriva dalla situazione storica dell'ultimo periodo dei cavalieri a Malta, si possono considerare facilmente in rapporto ad altri desideri che sia la Zauli Sajani sia gli esuli nutrivano per la causa maltese, tutti conducenti al risorgimento popolare e all'affermarsi della nuova nazione. Assumendo un tono classicheggiante, caratteristico degli scrittori dell'epoca, l'autrice si indirizza ai maltesi ed esprime la speranza di una vittoria imminente: "Malta, prediletta figlia del Mediterraneo, ultimo e sacro sasso d'Italia, io ti saluto. Riposa, riposa, ancora poche ore - se già non ti turba il sogno dello spavento. Verrà domani il sole ad illuminare la tua marina, i tuoi porti, le tue città..."

Anche il discorso che mette sulle labbra del patriota maltese Don Gaetano Mannarino è colmo di spirito di ribellione e ricorda le aspirazioni dei profughi italiani e dei loro collaboratori maltesi: "Quanto a te, soldato dei cavalieri, io ti compiango; tu hai mangiato del loro pane, ed hai giurato di combattere i loro nemici; la tua vita è venduta, tu non puoi fuggire la faccia di traditore; ma questo popolo è libero perchè Dio lo ha fatto libero, e distrutto l'Ordine, in questo popolo, in questo popolo solo è il diritto di scegliere il suo re. Figli di Malta, io veggo che non sono da voi lontani tempi di felicità, tempi di gloria.» Il riferimento storico ci porta indietro nel 1798 , quando i maltesi, in seguito al loro insorgere contro i francesi a causa della caduta dei Cavalieri, pensavano di proclamare il re delle Due Sicilie come il re di Malta, o di entrare a far parte dell'impero inglese. Conseguentemente, il giornale reazionario "L'osservatore maltese" lanciò un altro attacco, e definì il romanzo «sciocco (...) un insulto il più impertinente fatto all'onore nazionale dei maltesi" ciò non toglie che, dall'altro lato, questo spirito ribelle cominci ad evolversi tra il nuovo nucleo di liberali maltesi.

La posizione presa dall' “'Osservatore maltese” rispecchia soltanto la mentalità di una parte della popolazione; l'influsso degli esuli cresceva, e si comprendeva meglio la loro causa e la sua rassomiglianza alla condizione politica attuale della colonia

31. II ritorno dell'emigrato, Malta, Stocker Bros. \& Co., 1842, p. 9. 
maltese, così che cominciavano a manifestarsi di più sia la simpatia che 1'adesione. Il giornale, purtroppo, rimane il portavoce dei non-aderenti e degli anti-liberali. La poesia anonima che segue indica chiaramente da quale punto di vista erano giudicati gli esuli dai direttori della pubblicazione:

\author{
Ritratto de' Carbonari \\ Capri barbati eroi, de il volgo ignaro \\ dice talor che nulla avete fatto, \\ la storia ha pronto penna e calamaro, \\ e lascierà di voi giusto ritratto. \\ Dirà che avete in pochi giomi sfatto \\ quel che secoli molti edificaro; \\ e proveran le chiose al volgo matto \\ che sfugger tutto è un operar preclaro.
}

Dirà Ie lingue in bestemmiar valenti, dirà le mani agli assassini pronte, dirà le gambe allo scappar correnti.

No, non v'ha studio, non età che basti a cancellar dall'onorata fronte questa e mill'altri gloriosi fasti. ${ }^{32}$

Il giornale non mancò neanche di informare il pubblico delle sue opinioni intorno al pericolo dell'infiltrazione dei profughi nel crogiuolo della vita del paese. In una serie di articoli cominciò ad inserire la parola 'liberalismo' e ad identificarla con loro e con il gionale più rappresentativo della loro attività, "Il Mediterraneo". ${ }^{33}$ A suo giudizio, la Chiesa cattolica doveva intervenire con tutta la sua forza contro la diffusione degli esuli fra le famiglie: "Può ella, la chiesa, guardar con occhio tranquillo la distruzione non che del Cattolicesimo e del Cristianesimo, ma perfino d'ogni religione ancorché naturale? Può egli, lo stato, restare indolente al vedersi minare sordamente ogni principio di ordine e di dipendenza? E parlando particolarmente di noi maltesi, che siamo cattolici, vogliamo noi distruggere quel governo che noi stessi abbiamo scelto? Che abbiamo noi che fare colla "Giovine Italia"? Perdere forse la fede ed il costume

32. “O. M.”, 7/12/1842, p. 80.

33. Cfr. i seguenti articoli: II liberalismo in Malta ossia "II Mediterraneo" e compagni, 7/2/1842, pp. 78-79, 24/12/1842, p. 93; I liberali in Malta, 5/7/1843, pp. 199-201; La filosofia del "Mediterraneo", 5/7/1843, pp. 198-199. 
(...). Dal consorzio di cotesti signori della "Giovine Italia" non apprendiam' altro, se non che l'impietà, la scostumatezza, la fellonia ed il disprezzo d'ogni legge umana e divina. Purtroppo lo sanno quelle famiglie, le quali piangono di aver aperte le proprie case, e peggio d'aver affidato i propri figli... ${ }^{34}$

Se si elimina questo elemento reazionario, si conclude con facilità che la presenza degli esuli non soltanto pervadeva la vita culturale del paese ma iniziava attivamente lo spirito dei maltesi all'ideale della libertà e dell'autonomia nazionale. In ciò consisteva, dopo tutto, la sostanza contenutistica della letteratura risorgimentale italiana.

\section{La stampa libera}

L'avvenimento più importante della prima metà dell'Ottocento nella vita interna dell'isola, fu la concessione dal governo inglese della libertà di stampa nel 1839. Sotto il governo britannico, la stampa fu rigidamente regolata, e le richieste dei maltesi per impiantare tipografie furono respinte. L'unica tipografia che esisteva era quella del governo. Un permesso, negato ai cittadini locali, era concesso solamente ad alcune associazioni religiose e alla società missionaria della chiesa anglicana che pubblicava libri religiosi destinati ad essere distribuiti nel Mediterraneo, nell'Adriatico e nel Medio Oriente. ${ }^{35}$ Questo diritto fu concesso dopo vari appelli dai maltesi ${ }_{3}^{36} \mathrm{e}$, malgrado l'opposizione di governi stranieri che erano del tutto contrari alla diffusione della parola stampata nell'isola, la loro preoccupazione non era infondata; il vivo nucleo di esuli italiani era determinato a servirsi della stampa, mentre la scontentezza sociale e politica del popolo poteva facilmente trovare il veicolo adatto ad esprimere la propria insoddisfazione e ad organizzarsi contro la prepotenza straniera.

Gli ambasciatori di governo di varie nazioni che non volevano concedere una costituzione liberale, quale l'Austria e le Due Sicilie, insistevano ripetutamente con il governo britannico a non acconsentire alla volontà popolare, soprattutto perchè temevano che i ribelli potessero utilizzare la stampa maltese per divulgare i loro ideali. Il Vaticano scrisse anche al vescovo F. S. Caruana invitandolo a combattere la

34. Società segrete, 28/7/1843, p. 233; cfr. anche Stampa carbonaresca in Malta, ibid., pp. 251-252.

35. K. Sant, It-traduzzjoni tal-Bibbja u l-ilsien Malti 1810-1850, Malta, Royal University of Malta, 1975, p. 10 sgg.

36. Sono da sottolineare particolarmente gli interventi del patriota maltese Ġorg Mitrovich che ai primi di luglio del 1835 si recò a Londra a difendere la causa del suo popolo, e a mettersi in contatto diretto con il Gabinetto britannico. Cfr. The claims of the Maltese founded upon the principles of justice by George Mitrovich, a native of Malta and a faithful subject of the Crown and Great Britain, London, Mills \& Son, 1835. 
libertà di stampa, vedendo la probabilità che i profughi italiani, molti dei quali erano anticlericali (come si può dedurre immediatamente se si leggono $i$ loro scritti su diversi giornali maltesi) o massoni, potessero infiltrarsi nell' isola e oltre. L'opposizione venne anche dal Piemonte, governato dal re della Casa Savoia, che temeva che la stampa cadesse nelle mani degli italiani repubblicani a Malta. ${ }^{37}$ Come effetto della ricerca svolta dalla Commissione Reale costituita dal governo inglese nel 1836, furono inviati nell' isola due commissionari, John Austin e George C. Lewis, con lo scopo di condurre un'inchiesta generale e far diminuire la tensione politica. Nel 1838 presentavano un rapporto dettagliato al governo di Londra che, finalmente, concesse la richiesta libertà, con una ordinanza del 15 marzo 1839.

L'agitazione politica toccò un vertice e l'informazione fornita dalle inchieste dei due commissionari Austin e Lewis mise in chiara luce la situazione critica in cui si dibatteva la vita dell'isola. ${ }^{38}$ I due funzionari dovevano anche esporre il loro giudizio intorno alla possibilità che la stampa venisse sfruttata dagli stranieri con scopi politicamente controversi. ${ }^{39}$

In un Real Rescritto, comunicato al ministro degli affari esteri del Re di Napoli al ministero della polizia il 16 aprile 1839, si vede il disappunto del Re di fronte a questa concessione; e anche la piena consapevolezza del movimento liberale che si stava svolgendo nel paese: «Appena ne fummo informati non ne dissimulammo le tristi conseguenze per I'Italia e massime per i Reali domini, ove mai la funesta concessione avesse avuto effetto. E, rilevandole all'Inghilterra, impiegammo tutti i possibili mezzi per dissuaderla a divenirvi, ma le nostre speranze andarono fallite. Questa spiacevole e pericolosa occorrenza ha richiamato la seria attenzione del $\operatorname{Re}$ signor nostro. Sua maestà ha considerato potere essere la stampa libera accordata ai maltesi perniciosissima a questo regno, per la sua vicinanza a quella isola, quando gli scritti che colà vengono in luce s'introducessero. Ha riflettuto il $\mathrm{Re}$ che, quantunque pene vengano minacciate nella notificazione all'uopo dal Governo locale emanata contro gli abusi della stampa, queste però, oltre ad essere assai miti, non prevengono il male che si teme, nè sono dirette ad impedire che massime sovversive e liberali compariscano nei fogli maltesi. E non e meno da attendersi che aumentate le stamperie, vi si pubblichino articoli perniciosi, i quali attacchino i giomali monarchici sotto date di altri paesi .... La Maestà Sua quindi nel Consiglio ordinario di

37. H. Ganado, Rajt Malta Tinbidel, I, Malta, ll-Hajja, 1974, pp. 112-113.

38. Cfr. J. Austin - G. Cornwall Lewis, Reports of the Commissioners appointed to inquire into the affairs of the island of Malta, Malta, Government Press, 1838 . .

39. Cfr. Report on the expediency of introducing into Malta a liberty of printing and publishing, Malta, Government Press, 1838. 
Stato del 13 del corrente mese di aprile, uniformandosi al mio parere, ha ordinato di esercitarsi la più severa e rigorosa sorveglianza su tutte le provenienze da Malta, affin d'impedirsi la introduzione nel Regno degli scritti che si stampano in quell'isola, e particolarmente sui vapori. ${ }^{40}$

Il numero delle stamperie aumentava in breve tempo, particolarmente nella Valletta, e si cominciavano a stampare libri e giornali tanto per i maltesi quanto per gli stranieri e a inviarli clandestinamente, in diverse parti della penisola, soprattutto nella Sicilia. "Lo spettatore imparziale" ebbe inizio il 23 aprile 1838, diretto da Francesco Panzavecchia; il permesso fu concesso dal Governatore Bouverie, poco prima che si approvasse la legge, con lo scopo di diminuire l'agitazione politica. ${ }^{41}$ II 12 maggio dello stesso anno uscì il "Portafoglio maltese", diretto dall'avvocato Paolo Sciortino, un politico di tendenze moderate e conservatrici. Fra i giornali che dominavano l'opinione pubblica, e che davano un grande contributo alla cultura e alla nuova presa di coscienza nazionale - sempre nei limiti dei rapporti italo-maltesi-c'era "Il mediterraneo", fondato, come si è visto, dallo Zauli Sajani e da Carlo Cigognani Cappelli, e pubblicato tra il 1838 e il 1874. Molti dei profughi e poi diversi liberali maltesi che s'identificavano con il movimento italiano e cercavano di intravvedere l'ispirazione ad un movimento analogo maltese, collaborarono anonimamente al giornale, ma fino ad un certo periodo ne fu responsabile soltanto lo Zauli Sajani che spesso analizzava la situazione politica della patria e la vita sociale e culturale di Malta. La "Giovine Italia", intuendo che la concessione della libertà di stampa era imminente, si preparava ad inviare macchinari e apparati tipografici necessari, ${ }^{42}$ e presto pubblicò una grande quantità di fogli contenenti attacchi contro il Re di Napoli e li diffuse fra il popolo. ${ }^{43}$

Nell'aprile 1839 prendeva dimora nell'isola Salvatore Costanzo, spedito dal Governo di Napoli come emissario incaricato di far uso della stampa con 1 'intento di costringere l'Inghilterra a revocarne la concessione. Ma appena arrivato, Costanzo, insieme con Salvatore Tornabene, fondò un foglio, "II Corriere maltese", che, a causa degli attacchi scagliati contro il Re di Napoli e la sua famiglia, svelò in poco tempo lo scopo reale dei due scrittori. ${ }^{44}$ Costanzo, scrittore teatrale, si dedicò attivamente al giornalismo e fondo' un secondo giornale, "l'Aristide". La sua convinzione

40. Riprodotto da E. Gentile, Fonti documentali degli Archivi napoletani - Malta nelle carte di polizia dall83l al1847, “A. S. M.”, XI, fasc. III, 1940, pp. 244-245.

41. A. V. Laferla, op. cit., p. 166.

42. B. Fiorentini, op. cit., pp. 37-53.

43. H. Ganado, op. cit., p. 114.

44. B. Fiorentini, op. cit., p. 47. 
rivoluzionaria, è evidente dalla definizione che dà di Lammennais: "Lammennais nella Schiavitù moderna, nel Libro del popolo ed in altre sue opere, sempre difende i diritti dei meschini dispotizzati, e grida contro l'arbitrarietà de' tempi. ${ }^{45} \mathrm{Il}$ Costanzo, anzitutto, cercò di propagare a Malta il sentimento ribelle di cui era animato quando nel 1841 pubblicò il libro Ragioni che mi spinsero ad emigrare dal proprio paese.

Erano numerose le gazzette, scritte prevalentemente in italiano, e anche in inglese e in maltese. ${ }^{46} \mathrm{Il}$ giornalismo era destinato a far crescere l'interesse culturale e politico, e l'antico legame tra Italia e Malta poteva assumere nuove dimensioni; gli scrittori e i giomalisti aumentavano e si trovavano in grado di collaborare agli stessi ideali che, con il passar del tempo e con l'aggravarsi della situazione prima in Italia e poi nell'isola, andavano prendendo la forma di causa ed effetto. Il tono, il coraggio e il grido appassionato del patriota Mitrovich risentono dell'ansia del messaggio mazziniano: "Il tempo della persecuzione e passato. Levate dalla vostra mente ogni minima ombra di timore, perchè si tratta di ricorrere ad un'assemblea di un popolo libero, che vi dà piena facoltà di parlare apertamente, domandare e ripetere. Ora è il momento, miei cari fratelli, e non dovete perderlo (...). Siate certi che verra` un giomo che il popolo maltese sarà reso felice, sarà liberato dalla sua schiavitu’, ben trattato e accarezzato. II tempo della nostra rigenerazione si avvicina". ${ }^{47}$

Non era ancora il momento per fare emergere il concetto di un paese totalmente indipendente, ma la parola 'rigenerazione' è già un passo nella giusta direzione, molto vicina a risorgimento. Come i giomalisti italiani nell'isola gridavano con toni eloquenti, così i patrioti maltesi cominciavano a trovare occasione, mediante la stessa stampa sfruttata dagli esuli, a pronunziarsi e a suscitare le masse a diventare consapevoli dei loro diritti. Lo spirito profetico del Mazzini non era del tutto assente negli scritti del Mitrovich: "La nazione dovrà presto trionfare, la riuscita di una causa nobile e giusta, com'è la nostra, è certa; un altro poco, e vedrete". ${ }^{48}$ Il sogno mazziniano, piuttosto comune più tardi nella poesia patriottica locale, faceva parte del nuovo ideale. Una parte della popolazione simpatizzava apertamente con gli esuli, anzi senza la collaborazione di vari elementi locali sarebbe stata praticamente impossibile

45. S. Costanzo, II paese e il governo di F. Lammennais, trad. dal francese, con prefazione e note, Malta, Izzo; 1841, p. IV.

46. Tra il 1838 e il 1870 f urono pubblicati circa 180 giornali (cfr. V. Bonello - B. Fiorentini L. Schiavone, Echi del risorgimento a Malta, Malta, Comitato della Società Dante Alighieri, 1963, pp. 30-110.

47. G. Mitrovich, Indirizzo ai maltesi da parte del loro amico, Londra, Mills e figlio, 1835, pp. 14-15.

48. Ibid., p. 24. 
qualsiasi attività svolta dagli stranieri. Ad esempio, dei negozianti maltesi servirono da intermediari per introdurre in Sicilia opuscoli mazziniani e lettere di emigrati. ${ }^{49} \mathrm{Il}$ maltese più noto fra i giovani che s'identificavano con la causa degli esuli era Emilio Sciberras, amico tanto di Giuseppe Lamberti, segretario della congrega centrale della "Giovine Italia", che corrispondevà regolarmente con lui tra il 1841 e il 1848, quanto dello stesso Mazzini. ${ }^{50}$

\section{Esuli letterati tra i più noti}

Francesco Orioli, professore di fisica nell'università di Bologna che nel 1831 era entrato a far parte del Governo Provvisorio della città, arrivò a Malta verso il 1840, e aspirò ad insegnare la lingua italiana all'università, ma la sua candidatura non ebbe successo, probabilmente a causa dell'opposizione della fazione antiliberale ${ }^{51} \mathrm{Nel} 1840$ pubblicò un libro di poesie, Versi, una raccolta di memorie di un poeta esule che trova il suo rifugio o nella confessione pacifica delle proprie sofferenze o nell'esortazione appassionata all'impegno patriottico. I due poli estremi, entrambi stati d'animo assai tipici della poesia d'esilio, sono intrecciati insieme a far emergere la visione di un poeta e di un combattente, dimensioni inseparabili di una sola identità. Quando il poeta è ispirato alla rivolta politica, la memoria prende gran parte:
Caduto in su la polvere
il serto raccogliete, 1 'antico acciar di Romolo
Itali, in man prendete sangue di mille bravi, la sua ruggine lavi, sorgete! vi scuotete! ${ }^{52}$

Dall'altro canto, si chiude in se stesso in momenti di riflessione rassegnata e produce versi semplici e saturi di una malinconia silenziosa:

49. B. Cellini, op. cit., p. 65.

50. Cfr. G. Castaldi, Lettere inedite di G. Mazzini a E. Sceberras, “N. R. S"., IV, fasc. I, 1920, pp.109-117.

51. L. Schiavone, op. cit. pp. 149-150.

52. Frammenti d'un guerriero, Versi, Malta; Tonna, 1840, p. 33. 
lo pur d'amore immenso

amo il natal mio loco, sento, quando vi penso, nascermi in petto un fuoco.

Mentre vi reco il piede, dice ognun che mi vede: ecco una rondinella.

Vuol così la mia stella. ${ }^{53}$

Lorenzo Borsini, di Siena, poeta satirico, giornalista, ex-prete, musicista e cantante, che a Napoli aveva pubblicato due giomali teatrali, "Globo" e "Vesuvio"54, giunse a Malta il 27 gennaio 1841, insieme con i suoi tre figli e diede inizio ad una intensa attività giornalistica e letteraria e insegnava italiano e francese in una scuola aperta da lui stesso nella Valletta. Di tutto il suo contributo sui giornali, il più significativo è la fondazione di "Eraclito" (1842), di cui uno degli scopi era di combattere Don Giuseppe Zammit, detto 'Brighella', un poeta satirico maltese dalla vena aspra. Il liberalismo e il coraggio del Borsini, costruiti sulla visione nazionalistica del secolo, che vedeva nella patria il centro degli ideali umani, salgono alla superficie in questo brano: "Prima si nasce uomo, e poi si diventa cittadino. E chi non sa esser uomo è degno di abitar tra le fiere, a cui la patria è un nome vuoto di senso. Amare prima Dio, e poi la patria - ecco la scala (...). Ogni altro ordine è anarchia, ogni altro sistema è caricatura. ${ }^{55}$ Le sue opinioni e le simpatie che nutriva apertamente nei suoi scritti a favore dell'emancipazione costituzionale dei maltesi, costrinsero il Governatore O'Ferrall a mandare un dispaccio contro di lui al ministro delle colonie affinché lo mandasse fuori di Malta. La sua ansia per l'unificazione della patria e il suo antagonismo contro l'amministrazione britannica nell'isola, caratterizzano una parte della sua produzione giomalistica sullo "Stenterello" (1845-1871) e della sua opera letteraria. ${ }^{56} \mathrm{Il}$ Borsini pubblicò un numero considerevole di libri a Malta: Parole poche (1841), II sacrificio de' miei baffi - poemetto eroicomico ${ }^{57}$ (1842), Viaggio sentimentale al camposanto colerico di Napoli (1842), La spia - commedia in tre atti (1843), L'asino - canti dodici, libera traduzione (1844), ${ }^{58}$ Il novissimo galateo

53. II ritorno dell'esule, op. cit., p. 9.

54. "Med.", 8/8/1838, p. 10.

55. Notandum ad un notandum, "Med", 4/10/1848, p. 13.

56. G. Mangion, op. cit., pp. 32-33.

57. II poemetto fu recitato dal poeta stesso nel Real Teatro di Malta, la sera del 25 aprile 1842.

58. In questo poema descrive, con uno stile schietto e agile, il Regno delle Due Sicilie e il rifugio concesso agli esuli italiani in Microscopoli, un altro nome con cui si indjcava Malta. 
(1851). Durante lo stesso periodo collaborava anche con i giornali "Aristide", "Il mediterraneo" e "La speranza".

Gli esuli cominciavano ad affluire in grandi gruppi dopo la Restaurazione, e particolarmente dal 1849 in poi. Tra il 1848 e il 1860 Malta diventò un centro importante per i rifugiati che, falliti i moti nella penisola, cercavano scampo nel paese vicino. Benchè nei primi decenni dell'esodo arrivassero vari personaggi di rilievo, fra i quali Luigi, Nicola e Paolo Fabrizi, ${ }^{59}$ i fratelli Bandiera, il loro apporto non fu grande come lo fu più tardi. Nel secondo periodo, il numero aumentò enormemente, e spesso si trattava di gente che aveva varie possibilità di agire e di partecipare allo svolgimento del destino nazionale; di conseguenza, la loro influenza sulla piccola comunità maltese fu più profonda. A volte i profughi, arrivati nel porto, non erano ammessi nell'isola, e tale decisione suscitava malcontento e proteste. Tuttavia, nel presente periodo arrivarono Pasquale Calvi, Matteo Reali, Ruggero Settimo, Francesco De Sanctis, Adriano Lemmi, Francesco Crispi, ancora Nicola Fabrizi, Daniele Manin, Guglielmo Pepe, Rosalino Pilo e la principessa Trivulzio Belgiojoso. ${ }^{60}$

Nel gennaio 1848 vi fu l'arrivo di Luigi Settembrini, ${ }^{61}$ autore della Protesta del popolo delle Due Sicilie (1847), Lezioni di letteratura italiana (1866-1872), Scritti vari (1879), traduzioni di Luciano e soprattutto di un'opera piena di informazione politica e di sentimento patriottico ed umano, le Ricordanze della mia vita (1879), in cui parla del suo soggiorno a Malta: «La prima cosa che mi colpì in Malta fu leggere per tutte le cantonate grandi avvisi di vendita di mobili di Don Carlo di Borbone principe di Capua. Mi fece pena anzi dolore a vedere uno dei reali di Napoli cosi vituperato, e ne domandai al dottore, il quale mi rispose: Muore di fame, e non puo` uscire di casa, se no i creditori l'arrestano (...). Subito mi trovai in mezzo agli esuli, e li conobbi tutti. Agostino ed Antonio Plutino di Reggio, Carlo Gemelli di Messina con altri messinesi che avevano fatto a le schippettate il primo settembre (...) e tra molti altri di cui non ricordo i nomi, Lorenzo Borsini, toscano, che era piacevole poeta, ed aveva fatto il prete, il tabaccaio, il cantante, e in Malta faceva l'occhialaio, e aveva due figliuoli, e io andava sempre a la sua bottega per udirlo parlare .... Talora andava dal Gemelli che era un colto e gentile uomo di lettere, ed era in letto per malattia, e gli venivano

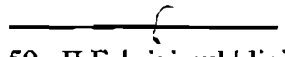

59. II Fabrizi pubblicò diversi libri nell'isola.

60. B. Cellini, op. cit., pp. 62-63.

61. La data del suo arrivo risulta da quello che scrisse nelle Ricordanze, ma questa deve indicare un suo secondo soggiorno nell'isola. Secondo L. Manzi, I senatori Agostino e Antonino Plutino profughi a Malta nella rivoluzione del 1847 a Reggio Calabria, "A.S.M.", III, fasc. I-IV, 1932, p. 176, il Settembrini era a Malta alla fine del 1847 , e fu presente ad un pranzo dato in onore dei fuggiaschi. 
intorno gli altri siciliani che gridavano come ossessi e tempestavano parlando della rivoluzione di Palermo. ${ }^{62}$

Luigi Fabrizi è noto soprattutto per la poesia A generosi maltesi - gli esuli pubblicata net 1848 . Avendo vari versi ripetuti come ritornello, la canzonetta ha un'impostazione musicale e si svolge su melodiosità ritmiche che s'intrecciano alle ripetizioni, ai parallelismi, al ritmo danzante dei settenari, creando cosi l'effetto voluto di inno entusiastico e trabocchevole. Il poeta esprime la sua soddisfazione per trovarsi nell'isola, "terra d'Italia", dove il pensiero è fraterno e cessa l'esilio. Il motivo principale dice che Malta è figlia d'Italia e fra poco si unirà con la "madre patria"; evidente anche l'esortazione del Fabrizi ai maltesi perchè si ribellino e combattano per i loro diritti fondamentali:

Il cielo propizio

o terra diletta

ti renda alla Madre

che sorge e t'aspetta

fra l'Itale squadre

ritorna a pugnar.

Risorge l'Italia!

E nostro il futuro

n'è pegno di gloria,

n'è pegno sicuro

l'antica memoria

d'Italia il valor.

Melito d'Ausonia

tu fervida terra,

tu palpiti e godi

al grido di guerra

agogni coi prodi

divider l'onor.

Nello stesso periodo partirono per la Sicilia i fratelli Agostino e Antonino Plutino che erano fra i responsabili piu` importanti dell'insurrezione di Reggio nel

62. Opere scelte, a cura di L. Negri, Torino, Unione Tip. Editriece, 1955, pp. 202-203. II Settembrini ricorda anche che a Malta si poteva leggere qualsiasi scritto della "Giovine Italia", e non soltanto il giomale dallo stesso nome, ma pure lo statuto della setta. 
settembre 1847 e che si salvarono dalla condanna di morte con il rifugiarsi nell' isola. Nel febbraio 1848 tre esuli siciliani, Mirone, Fatta e Piazza arrivarono con l'intento, dato loro dal Comitato Rivoluzionario Palermitano, di acquistare 2.000 fucili inglesi. Approdarono anche altri ben noti reazionari napoletani che furono costretti a fuggire dalla rabbia del popolo, fra i quali il maresciallo Del Carretto, Mons. Celestino Cocle, arcivescovo titolare di Patrasso e confessore del Re Ferdinando. Pochi giorni dopo giunsero a Malta 49 gesuiti, la cui presenza fu la causa di una grande lotta fra liberali e conservatori. C'era nel gruppo padre Carlo Maria Curci, noto come letterato, e padre Matteo Liberatore, professore di filosofia. Il 25 maggio 1848 arrivò un altro gruppo di esuli napoletani, tutti profughi della rivoluzione, tutti deputati calabresi che avevano ricoperto un ruolo preminente nell' insurrezione avvenuta a Napoli dieci giomi prima. ${ }^{63}$

\section{Nascita del Movimento Liberale}

Il 1 luglio 1848 il Governatore O'Ferrall mostrò la sua profonda preoccupazione riguardo la presenza degli esuli e inviò un dispaccio importante all'Ufficio Coloniale di Londra. ${ }^{64}$ Mentre a Malta si progettavano diverse riforme fondamentali, l'elemento radicale sparso nelle varie sezioni della popolazione apparve pericoloso. Tanto i maltesi quanto l'amministrazione inglese cominciavano a sentire finalmente il bisogno di formulare una costituzione migliore; l'insoddisfazione pubblica si andava facendo più chiara e insofferente. Lo scetticismo dei liberali italiani, dei patrioti e dei simpatizzanti maltesi si faceva sentire sempre di più sulle colonne del "Mediterraneo", e la critica lanciata contro il Governatore si faceva più severa. ${ }^{65}$ La presenza degli esuli continuava a mettere in rilievo noti personaggi. Tra giugno e luglio $1848 \mathrm{ci}$ fu il cardinale Gabriele Ferretti, segretario dello stato pontificio, arcivescovo di Napoli. Negli stessi mesi dimorò nel paese il principe Ferdinando Carlo di Borbone, duca di Lucca e più tardi di Parma. Contemporaneamente lasciò l'isola il principe Carlo di Capua e nel settembre 1848, dopo il bombardamento di Messina, giunse un gruppo considerevole di messinesi; in quel mese moriva a Malta Paolo Lo Uzzo dopo aver subito gravi ferite nella resistenza di Messina. ${ }^{66}$

Il 1 ottobre 1848 si fondò una società letteraria nota come il "Circolo maltese", ${ }^{67}$ composta di circa un centinaio di persone, maltesi e residenti stranieri, tutti membri della classe colta. Secondo il programma promulgato sui giornali, lo scopo era di

63. G. Mangion, op. cit., PP.' 22-30.

64. Ibid., p. 31.

65. Cfr., ad esempio, "Med.", 25/10/1848, passim.

66. Riguardo i funerali del Lo Uzzo, cfr. "Med,",4/10/1848, p. 13.

67. G. E. Zarb, Circolo maltese, "Med"., 4/10/1848, p. 13. 
coltivare le lettere in quanto esse tendono a produrre un miglioramento nelle bisogne intellettuali, morali ed economiche del popolo; tenere gli studiosi a giorno delle scoperte e de' perfezionamenti che nelle arti quotidianamente si succedono, procurare una piacevole ricreazione alle menti travagliate dalle cure diurne. II "Circolo maltese", fondato su proposta di Nicola Fabrizi ${ }^{68}$ è chiamato dalla stampa antiliberale una "fredda, meschina e pigmea copia de' circoli italiani", ${ }^{69}$ aveva il suo centro al numero $157 \mathrm{di}$ strada mercanti, Valletta, ${ }^{70} \mathrm{e}$ fu frequentato anche da molti politici emigrati; i quattro fratelli Emilio, Tancredi, Ruggero e Pelagio Sceberras, nobili maltesi e attivissimi nell'ambito politico, usavano confortarli, aiutarli e farsene garanti nei confronti della polizia locale. Il circolo dava una spinta anche alla composizione di poesie estemporanee. Soleva organizzare accademie letterarie durante le quali alcuni poeti, italiani e maltesi, improvvisavano versi d'occasione. ${ }^{71}$ Questo rapporto fra comitati rivoluzionari maltesi e altri gruppi italiani, seguitò a svolgersi per interi decenni. Ad esempio, nel 1854 G. Vollaro, da Tunisi, venne a Malta per organizzare una spedizione a Palermo; alcuni esuli mazziniani lasciarono l'isola con una speronara maltese e sbarcarono a Roccalumera, a sud di Messina, con lo scopo di sollevare la popolazione; oltre i fratelli Sceberras, assistevano gli esuli anche Vincenzo Fenech, G. Balbi e G. Camenzuli. ${ }^{72}$

L'elemento liberale e rivoluzionario cominciò a identificarsi anche con associazioni create dai maltesi e frequentate dagli esuli. Si potevano vedere, affissi qua e là, manifesti a favore delle scuole per l'infanzia; le vetrine dei negozi erano piene di diverse opere pubblicate a Livorno, di Dante, del Manzoni, del Nota, deI Pellico, del padre Soave, e altresi traduzioni dall'italiano. ${ }^{73}$ Silvio Pellico suscitò simpatia con Le mie prigioni, e le tragedie del Guerrazzi e del Nicolini divulgavano lo spirito di libertà e di odio contro la tirannide, mentre il Primato, $i$ Prolegomeni e il Gesuita moderno del Gioberti, le Speranze d'Italia del Balbo e la Protesta del popolo delle Due Sicilie del Settembrini e altre opere furono lette da molti. La propaganda personale

68. B. Fiorentini, op. cit., p. 148.

69. “ “0”., 31/5/1851, p. 735.

70. Cfr. "Med.", 4/10/1848, p. 13.

71. Cfr. "Av". 23/2/1850, p. 104.

72. E. Rossi, op. cit., pp. 59-60.

73. G. Bonifacio, Un piemontese a Malta cent'anni addietro,"A. S. M". , XII, fasc. III, 1941, p. 147. Gaetano Fil. Baruffi, il piemontese di cui scrive il Bonifacio, probabilmente si riferisce alle scuole private aperte nell'isola dagli esuli. Cfr. anche H. Ganado, op. cit., p. 112. Per una simile descrizione di Malta, particolarmente riguardo i piani ideati dai ribelli maltesi per una costituzione liberale, cfr. N. William Senior, Conversations and journals in Egypt and Malta, II, London, Sampson Low \& Co., 1882, p. 254. 
e la stampa dei profughi, la loro condotta esemplare e le loro sofferenze suscitavano un vivo interesse nella loro causa e simpatie per i loro problemi. ${ }^{74}$

La letteratura, benchè scritta da una classe ristretta di uomini di cultura, si richiamava al popolo e, malgrado non fosse apprezzata da tutti, essendo scritta in italiano, era causa di prestigio e di stima per i letterati. Lo sbarco a Malta di qualche poeta risorgimentale italiano era sempre, infatti, un'occasione per mettere in evidenza l'aderenza di un giornale al concetto di relazione reciproca tra letteratura e sviluppo politico. Ad esempio, l'arrivo del poeta Giuseppe Regaldi fu un'occasione simile per il Portafoglio maltese. Il poeta sbarcò il 15 novembre 1849 e il giornale scrisse: "Abbiamo il piacere di annunciare l'arrivo, in questa isola, del poeta estemporaneo Giuseppe Regaldi, scampato da Napoli alle persecuzioni di quel Governo. La fama che questo alto genio si è acquistato in poco tempo, in Italia non solo, ma pure in Francia, ove Lamartine e Victor Hugo gli resero omaggio di loro ammirazione, fama di cui fra noi fu preceduto il poeta, vale più che qualunque cenno potremmo noi fare dei suoi celebri componimenti. Questo estemporaneo, se non supera tutti gli improvvisatori viventi, non è secondo ad alcuno. Basta dire che fanno chiara menzione di lui $i$ più severi giudici che vanta oggi la letteratura italiana. Romani, Brofferio, Sacchi, Mauri, Fornaciari, Cibrario, tutti ammirano l'ingegno del Regaldi. Noi speriamo che il celebre poeta non ci negherà l'opportunità di sentirlo ed ammirarlo, come 1 'hanno sentito ed ammirato le prime città d'Italia e di Francia". ${ }^{75}$ Aderendo alla richiesta dei maltesi, il Regaldi, noto a Malta come "l'ultimo bardo d'Italia", ${ }^{76}$ tenne un paio di accademie poetiche. Il 26 dicembre, alla presenza di numerosissime persone, improvvisò dei versi su vari temi, fra i quali La battaglia di Novara, La Valette e'assedio di Malta del 1565, La mia vita e L'esule. Il 7 gennaio organizzò un'altra accademia poetica, e fu applaudito da oltre trecento ammiratori. Quando fece ritorno a Malta, nel 1853, improvvisò altre poesie in locali pubblici, come l'Università e la Biblioteca Nazionale. ${ }^{77} \mathrm{Il}$ Regaldi cominciò ad improvvisare i versi a Novara e poi in Francia. Una delle poesie che compose a Malta è Novara, una saffica scritta nel dicembre $1849 .{ }^{78}$ I1 tema dell'amarezza dell'esilio si mescola con 1'ansia della battaglia:

74. A.V. Laferla. Op.cit., pp. 200-201.

75. B. Fiorentini, op. cit., p. 154.

76. L. Schiavone op. cit. p.155.

77. B. Fiorentini op.cit. p.154.

78. Cfr. I poeti italiani del secolo XIX, III, a .cura di R. Barbiera, Milano, Treves, 1915, pp. 851-853. 
Pugnate, o figli del bel paese;

se amor vi sprona d'elette imprese, vi accenda all'ire di santa guerra

l'Itala terra.

Cercherò pace di fossa in fossa,

la 've del padre dormono l'ossa;

e d'ogni parte risponderanno

voci d'affanno.

Nell'ottobre 1849, per iniziativa di un gruppo di esuli, si fondò l' "Associazione per una mensile sovvenienza a Venezia”. Un gruppo di maltesi, fra i quali G. GrechDelicata, Enrico Naudi e Filippo Pullicino, organizzarono una commissione allo scopo di raccogliere donazioni, ${ }^{79}$ un modo utilissimo per aiutare i ribelli. Era appena fallito il tentativo del Piemonte per l'indipendenza nazionale. A Napoli il Re Ferdinando riuscì ad avvantaggiarsi del caos causato da liberali senza esperienza e riacquistò il suo potere. Nel maggio 1849, in seguito all'intenso bombardamento sulle città siciliane, cadde anche la Sicilia. Il Papa rinnovò il proprio potere nel luglio 1849 , e i moti di Napoli, Roma, Venezia e la Lombardia fallirono purtroppo anch'essi. ${ }^{80}$

I siciliani che avevano preso parte alla ribellione erano costretti a fuggire dal paese; oltre seicento patrioti sbarcarono a Valletta insieme con i loro dirigenti, fra i quali Ruggero Settimo, il presidente dell'antico governo. L'amnistia concessa dal Re di Napoli nel maggio 1849 invitava la maggior parte dei rifugiati a ritornare in Sicilia, ma alcuni dei capi a cui non fu concesso il perdono dovettero rimanere nell'isola ${ }^{81}$ Ruggero Settimo,principe de Fetalia, abitava in Valletta. Era stato un tempo ammiraglio della Sicilia e durante la rivoluzione del 1848 era stato eletto presidente del governo provvisorio. Nel 1861, quando ormai l'unificazione era fatto compiuto, fu eletto presidente della Camera Superiore del Parlamento italiano, ma mori nella capitale maltese nel $1863 .^{82}$

Fra i rifugiati che giunsero a Malta il 2 maggio 1849 c'erano lo storiografo Michele Amari e lo scrittore Michelangelo Bottari. Quest'ultimo era in contatto con il giovane liberale maltese G.B. Naudi che tentava di introdurre manifesti rivoluzionari

79. “Med.", 4/4/1849, pp. 12-13.

80. D. Richards,Op. cit., pp. 150-152.

81. T. Zammit, Malta - the Maltese islands and their history, $3^{\mathrm{a}}$ ed., Malta, A. C. Aquilina \& Co., 1971, p. 301.

82. Ibid., p. 323. 
pubblicati dal Mazzini e lettere di rifugiati siciliani a Malta, e fu espulso dal paese. ${ }^{83}$ Recatosi in Egitto, il Bottari ritornò poi a Malta e continuò la sua attività letteraria e politica. Compilò con Guglielmo Finotti "Il corriere mercantile di Malta", ma è noto sopratutto come l'auture di vari romanzi: Giorgio il pilota (tradotto in maltese e pubblicato da Anton Muscat Fenech nel 1880) Il Gran Maestro La Cassiere, La sposa della Musta, che prende lo spunto da una fiaba popolare maltese, e Giammaria, ovvero l'ultimo dei baroni Cassia, pubblicato nel 1857. Il Bottari scrisse quest'ultimo romanzo dopo aver condotto una lunga ricerca su documenti conservati nell'isola. Ambientato nel circuito ristretto dei nobili maltesi, particolarmente della famiglia Cassia, il romanzo ebbe un grande successo tra il pubblico maltese, così che fu pubblicato per la prima volta a puntate sul giornale "Malta" e poi Anton Muscat Fenech, scrittore in lingua maltese, lo tradusse per essere pubblicato su "Il-Habbar Malti". Più tardi fu pubblicato di nuovo sia in maltese che in inglese. ${ }^{84}$

O'Ferrall e i suoi ufficiali non potevano guardare i radicali di buon occhio. "Il mediterraneo", "Malta Mail", "Malta Times" e più tardi "L'avvenire" non vedevano altro che ingiustizia nell'amministrazione del governo civile. Le restrizioni di $O^{\prime}$ Ferrall e la direzione generale trovarono un'insoddisfazione su larga scala. ${ }^{85}$ II Governatore si oppose alla concessione immediata di maggiori poteri alla popolazione. In una lettera al Segretario di Stato scrisse che "whatever may be the opinion in regard to the advantage of change in other countries, none of the considerations which influence that opinion in an adverse sense is applicable to the Maltese". ${ }^{86}$

"qualunque siano gli argomenti sul vantaggio di una riforma in altri paesi, nessuna considerazione che influisce su quell'argomento in maniera avversa è applicabile ai maltesi”!

Ma l'entusiasmo patriottico che invase l'Europa, specialmente la penisola vicina, ebbe una eco a Malta, e la desiderata riforma fu parzialmente concessa con Lettere

83. B. Fiorentini, op. cit., p. 152.

84. Cfr. Te edizioni maltesi Giammarija Cassia jew inkella l-ahhar nisel tal-barunijiet Cassia grajja ta' Malta miktub bil-Malti mit-Taljan ta' M. A. Bottari minn Ant. Muscat Fenech,

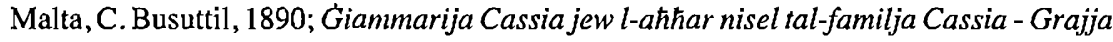
ta' Malta, trad. anonima, Malta, S. Paolo, 1923; l'edizione inglese Giammaria or the last of the barons Cassia - an historical romance of the 17 th century by $M$. A. Bottari, translated by Commander E. N. Price, R. N., Malta, 1923.

85. T. Zammit, op. cit., pp. 301-302.

86. Dispaccio del primo febbraio, citato da A. V. Laferla, op. cit., p. 204. 
Patenti dell' 11 maggio 1849; per esse fu istituito un nuovo "Consiglio di Governo", composto di diciotto membri; dieci di loro, compreso il Governatore, erano nominati fra i funzionari, e gli altri otto erano eletti in rappresentanza dal popolo. Le prime elezioni ebbero luogo nell'agosto 1849. Questa costituzione rimase in vigore fino al 1887 , quando il popolo maltese ottenne maggiori concessioni. ${ }^{87} \mathrm{Ma}$ le critiche e la scontentezza popolare cominciavano ad assumere maggiori dimensioni, grazie al contributo dei giornali e all'affratellamento dei maltesi con $\mathbf{i}$ loro amici stranieri. ${ }^{88}$

Il 12 maggio 1849 il Governo annunziò che intendeva adottare nuove misure per controllare rigidamente l'emigrazione politica. Presto si faceva nota l'espulsione di Luigi Zuppetta, affiliato alla "Giovine Italia", notevolmente conosciuto e stimato come scrittore dai maltesi ${ }^{89}$ Lo Zuppetta scrisse molto sui giornali, particolarmente su quelli fondati da lui stesso. Nel 1845 diede inizio a "L'Unione - gazzetta di Malta", compilata dai maltesi Nicola Zammit, ${ }^{90}$ G. Grech Delicata e Enrico Naudi, e in poco tempo si manifestò favorevole alla "rappresentanza popolare" dei maltesi. Nello stesso anno fondò e diresse " $I$ vagheggiatore delle scienze e delle lettere" includendo articoli contro il Re di Napoli. Un anno dopo cominciò la pubblicazione di "Giù la tirannide - voce di paese libero" una vasta raccolta dei suoi scritti era uscita sotto il nome di Raccolta de' migliori articoli legali e letterari. ${ }^{91}$ A causa del modo in cui influì sul pensiero dei maltesi, doveva lasciare il paese nell'agosto 1846 e andare a Londra, dove fu accolto dal Mazzini, ma ritornò nel 1847 dopo essere stato eletto deputato del Parlamento napoletano per Capitanata. ${ }^{92}$

87. E. Rossi, op. cit., p. 56.

88. A. V. Laferla, op. cit., pp. 214-215.

89. "Med.", 31/7/1844, p. 13.

90. Il Zammit (1815-1899) fu professore di filosofia, scrittore di opere letterarie, sociali, morali, filosofiche; e collaboratore importante della rassegna letteraria "L'arte", iniziata nel 1861. Per una completa bibliografia delle sue opere, cfr. R. Mifsud Bonnici op". cit., p. 557.

91. Cfr. L. Zuppetta, Raccolta de' migliori articoli legali e letterari, Malta, G. Grech e Co., 1848: un volume di letteratura patriottica, ricco di informazioni sulla vita politica in Italia e a Malta. Gran parte degli articoli sono saturi di citazioni da Dante, specialmente dall'Inferno e dal Paradiso, dal Petrarca, dal Rossetti, dal Berchet e dal Byron. A MaIta tenne anche diverse conferenze alle quali correva un grande pubblico di professionisti $e$ di letterati (cfr. "Med." 19/11/1845, p. II).

92. G. Mangion, op.cit., pp. 214-215 
Il 15 luglio 1848, 124 esuli romani entrarono in porto; e un giorno dopo un vapore greco arrivò con un gruppo di 53 da Civitavecchia. Più tardi, nello stesso mese, un altro gruppo di 87 profughi chiese il permesso di entrare, ma il Governatore rimase inflessibile nella decisione. II 21 luglio fu presentata una protesta collettiva, firmata da 48 maltesi, quasi tutti personaggi eminenti nella vita civile e culturale, ma non servì a niente. Le proteste pubbliche aumentavano, e certe reazioni venivano anche dall'estero, ad esempio dall'autore inglese Charles Dickens. Nel maggio 1849 si richiese il permesso alla polizia locale per lo sbarco di 238 esuli, quasi tutti soldati napoletani che avevano lasciato l'esercito del $\mathrm{Re}$, e siciliani che presero parte alla rivoluzione. Il governo britannico, su istruzioni del Governatore O'Ferrall, negò lo sbarco e l'opinione pubblica sbottò aspramente e reagì contro la decisione. L'arcivescovo Pubblio Maria de' Conti Sant esercitò la sua influenza per ostacolare lo sbarco, e pubblicò una lettera pastorale; dall'altro lato, "Il mediterraneo" scagliò un feroce attacco contro gli elementi anti-liberali del paese..$^{93}$

Questo cambiamento radicale nella mentalità maltese, o meglio la nuova disposizione ribelle, e, probabilmente, la causa che dentro il "Circolo maltese" (una società che non aveva soltanto, o soprattutto, un ideale letterario, ma piuttosto un intento politico diretto verso il problema costituzionale) si crearono due fazioni l'una contro l'altra; il gruppo radicale si staccò dal "Circolo maltese" per essere in grado di agire con libertà, e formò una nuova società, l' "Associazione patriottica maltese"..$^{94}$ L'opinione conservatrice continuava ad ammonire contro i pericoli degli intimi rapporti fra esuli e liberali maltesi. "L'ordine", apertamente contrario alla presenza degli italiani, li considerò come nemici dell'ordine costituito nell'isola e li accusò di avere lo scopo di istigare la popolazione ad insorgere contro la dominazione inglese e di identificare la loro causa con quella dell'isola. ${ }^{95}$

Il legame stabilito fra ribelli maltesi ed esuli italiani, in un periodo in cui Malta sembrava "una seconda Italia", ${ }^{6}$ si mette in evidenza anche con il fatto che alle adunanze segrete dell' "Associazione" presero parte attiva Giorgio Tamajo di Palermo, Ignazio Calona di Palermo, G. A. Nesci di Messina, Raffaele Lenza di Siracusa, Michele Orlando di Messina, Saverio Vollaro di Reggio, Giuseppe Oddo di Palermo, Raffaele Imbellone di Napoli, e altri italiani. ${ }^{97}$

93. Ibid., pp. 40-52

94. Ibid., pp.56-58

95. Cfr.,"O", 21/7/1849, pp. 11-12

96. A. Corbelli, Esuli italiani a Malta - dalle carte di S.M. il Re di Sardegna, "R.I." gennaiomarzo, 1929, p. 44.

97. Lettera di O'Ferrall a Lord Grey, 23/12/1850, citata da G. Mangion, op. cit., p. 68. 
Probabilmente essendo affiliata all" "Unità italiana" di Palermo, l' "Associazione" era in contatto con i comitati siciliani e, per mezzo del Tamajo e del Vollaro, svolgeva corrispondenza con patrioti italiani come Angelo Brofferio e Casimiro De Lieto. ${ }^{98}$ Lo stesso nome dell' "l'Associazione" ne ricorda altre simili esistenti contemporaneamente in varie parti dell'Italia. In quel tempo esistevano, ad esempio, I' "Associazione patriottica" di Roma, di Reggio, di Modena, di Genova, di Cagliari e di Napoli.99 "L'ordine" accusava ripetutamente l' "Associazione" di essere in continuo contatto con i gruppi rivoluzionari, a Malta e all'estero. Il sonetto anonimo che segue, dimostra come i dirigenti del giornale, e insieme a loro la parte conservatrice della popolazione, giudicavano l'"Associazione" maltese, a causa dei suoi ideali antibritannici e dei contatti che svolgeva:

Ciurma di saltimbanchi democratica, pitocca, miserabile, frenetica, ignorante, bestial, sciocca, salvatica, di un sudicio avvenir pseudo profetica.

Truppa di disperati empia, scismatica, irreligiosa, scellerata, eretica, piu' pestilente della lue asiatica, e puzzolente piu`della zaffetica.

Setta che chiesa e papa sprezza e critica, invidiosa, livida, malotica, piena il petto di bile antegesuitica.

Vile generazion, schiatta scriotica, ciurmaglia anticristiana, antipolitica; ecco l'Associazione patriottica. ${ }^{100}$

In verità, i rapporti diretti fra esuli e liberali maltesi esistevano da lungo tempo. I profughi fondarono nell'isola varie sette per sostenere la causa dell'unità. Nel 1843 sembra che a Malta siano esistite circa cinque società segrete di tale natura: "Liberi muratori", "Illuminati", "Carbonari", "Giovine Italia" e "Rigenerazione d'Italia".

98. B. Fiorentini, op. cit., p. 149.

99. Cfr. "O", 11/1/1851, pp. 568-569.

100. Ritratto dell'Associazione Patriottica, "O", 3/5/1851, p. 706. I particolari formali e la concezione complessiva del sonetto ricordano il bozzettismo poetico italiano che dipinge gruppi e persone, come fa, ad esempio, Arrigo Boito in Ad Emilio Praga, in cui traccia il bozzetto del gruppo degli Scapigliati. 
I profughi fecero anche un tentativo di dar vita alla "Giovine Malta", i cui membri dovevano essere maltesi. ${ }^{101}$

"L'avvenire", il cui messaggio era costruito sulla divulgazione popolare dell'istruzione e della cultura, si esprimeva spesso con uno spirito combattivo, ribadendo la libertà dei cittadini e del popolo maltese, e anzitutto il diritto della nazione di scegliere il proprio destino. Il suo compito era, evidentemente, di preparare le masse al momento della battaglia tra governo straniero e popolo: Il governare troppo fu d'ostacolo delle genti (...) fu il maggior nemico dei popoli, come non meno dei governanti medesimi. Con volere regolare tutto, e le arti ed il commercio si resero per lunga pezza improduttivi e l'educazione e lo sviluppo delle scienze furono ritardate (...). Se non che pare che tutta Europa debba risorgere dalla ben lunga malattia che ne fiaccava le forze - sembra che generalmente oramai si riconosca quanto illegittimamente siansi intervenuti i governi a danno della liberta dei sudditi. ${ }^{102}$

Il giornale cominciò presto ad essere atroce e severo contro l'amministrazione inglese, e l'ideale della libertà nazionale non tardò ad identificarsi con il duplice principio dell'opposizione all'autorità e dell'incitamento delle masse: "Un governo ipocrita, deciso a nulla fare che non fosse avverso al miglioramento del paese - deciso a soffocare lo spirito pubblico, a giovare l'ignoranza, e a scialacquare il denaro del popolo". ${ }^{103} \mathrm{Il}$ risorgimento che "Il mediterraneo" augurava e aspettava da un pezzo, adesso divenne la causa del nuovo giornale. Sarebbe inutile paragonare l'ideale dell' "Avvenire» con quello che il giornale della "Giovine Italia" amava ripetere: "Il popolo è ora risvegliato, e nulla lo potrà trattenere a farlo desistere, dal domandare ciò che ogni suddito britannico ha diritto d'avere - cioè una voce libera nell'amministrazione dei suoi propri affari. La gran cosa di cui si ha bisogno e la direzione. ${ }^{104} \mathrm{La}$ ricerca della direzione suggerita, sembra essere alla base della fondazione dell " "Associazione".

Nel 1851 si fondò un comitato mazziniano e nel marzo 1853 arrivò Francesco Crispi, ${ }^{105}$ appena espulso dagli Stati Sardi, con l'intento di impiantare una tipografia. Passò lungo tempo nello studio e frequentò gli archivi maltesi con il proposito di compilare una storia di Malta che mancava. A tale scopo dava inizio ad una serie di lettere allo storico Michele Amari. Da Malta strinse intime relazioni con Rosalino Pilo, con Giuseppe Mazzini, e con il patriota ungherese Kossuth. Nel 1854 fondò il giornale

101. B. Fiorentini, op. cit., p.103. La "Giovine Malta" fu` fondata piu` tardi, nel 1901.

102. "Av.", 12/111850, p. 49.

103. "Av.", 13/7/1850, p. 256.

104. La stampa di Malta, "Med.",15/11/1848, p. 11.

105. F. Crispi, Lettera dall'esilio, Roma, Tiber, 1918, p. 55. 
"La staffetta", che si occupò di problemi maltesi, ma soprattutto di questioni borboniche, e riuscì a penetrare in Sicilia e a Napoli. ${ }^{106} \mathrm{Nel}$ dicembre 1854 fu espulso dall' isola per aver scritto e pubblicato cose che non dovevano essere scritte da stranieri, ma continuò a corrispondere con gli esuli, particolarmente con Nicola Fabrizi. Nell' anno seguente pubblicò a Londra il volume Dei diritti della Corona d'Inghilterra sulla Chiesa di Malta, frutto di ricerche condotte nella Biblioteca della Valletta. ${ }^{107}$

Enrico Poerio, poeta drammatico, arrivò nel 1855. Tre anni dopo cominciò a scrivere articoli per alcuni giornali liberali e fece pubblicare il Saggio di teatro italiano. ${ }^{108}$ Il Poerio è anche il poeta della tragedia patriottica; una caratterizzazione sovrabbondante e un linguaggio retorico e aulico si mescolano insieme per creare situazioni e personaggi appartenenti alla storia nazionale, con l'intento di coinvolgere gli spettatori. In Beatrice Dalesmanno, scritta e pubblicata a Malta, il poeta esorta il popolo a ricordarsi della sottomissione nel passato e ad insorgere contro la tirannide:
È già gran tempo che soffriamo in muto libero sdegno. E' giunta l'ora alfine che lo sdegno favelli agli oppressori di libera vendetta e sanguinosa vive parole, che in più vivi fatti si trasfondan possenti (...).
Sapete voi se io covi
odio nel seno allo straniero, e quanto
approfittar vorrei della privata
offesa perchè venga al popol rotta
la vil catena che gli suona al piede.

Le vicende italiane del 1859-1860, specialmente le grandi vittorie di Garibaldi in Sicilia e nell'Italia meridionale, causarono un mutamento considerevole nell'emigrazione politica a Malta. Quasi tutti gli esuli che durante la cospirazione vi cercarono rifugio, partirono verso la patria per partecipare da vicino alla battaglia;

106. G. Ardau, Francesco Crispi, Milano, Ceschina, 1939, pp. 69-76. A proposito della parte che ebbe nella storia di Malta, Cfr. p. 83.

107. E. Rossi, op. cit., p. 60.

108. II titolo intero del libro e Saggio di teatro italiano, con l'aggiunta del poema di Beattie, "I piaceri della solitudine", è di quello di Mr Moore, "Pensieri notturni sulle tombe", tradotti dall'inglese per suo padre il tenente col. Leopoldo Poerio, Malta, Luigi Borg, 1858. 
al contrario, arrivarono a Malta molti funzionari borbonici, prima dalla Sicilia e poi dalle provincie meridionali e da Napoli. Ad un certo punto non furono molti, e rimasero inattivi, sperando nell'intervento delle potenze europee contro l'usurpazione piemontese a danno del Re Francesco II, ma quando s'accorsero che tutto questo non era possibile, s'agitarono, organizzarono conciliaboli, pubblicarono fogli e opuscoli e fondarono un partito con l'aiuto di alcuni maltesi. I rifugiati borbonici continuarono a fare propaganda per le loro rivendicazioni, e a diffondere scritti contro il governo italiano, e pubblicarono persino articoli reazionari sui giornali locali, ad esempio sul "Portafoglio maltese" e sul "Corriere maltese". Ma nel 1863 il loro numero si riduceva, e la loro attività veniva sempre più meno. ${ }^{109}$

\section{Garibaldi e Malta}

Il "Valletta" portò Garibaldi nell'isola il 23 marzo 1864; c'erano con lui otto persone, fra i quali i figli Menotti e Ricciotti. Quattro anni prima Garibaldi e i Mille giunsero a Marsala, presero Palermo e sconfissero i Borboni in tutte le parti della Sicilia, all'infuori di Messina. Poi attraversò lo stretto di Messina ed entrò a Napoli con grande successo. Tutto questo era ben noto al pubblico maltese. Le accoglienze che furono fatte al Generale avevano una dimensione nazionale; presero parte la stampa, la sezione più eletta della popolazione, la comunità inglese e i residenti liberali italiani. La stessa sera del 23 il "Corriere mercantile" pubblicò la seguente ottava che mette in rilievo l'accoglienza offerta dai maltesi:

Veder bramavi il popolo maltese?

Due soli giomi, e n'hai l'idea compita.

Pronto mai sempre a rovesciar le offese

contro chi a torto il suo disdegno incita,

calmo e severo, energico e cortese,

di cor bollente e sobrio nella vita,

ieri punisce il vile insultatore,

oggi con Garibaldi è tutto cuore.

"Il mediterraneo", "Malta Times and United Service Gazette", "The Malta Observer" e altri fogli gli porsero una accoglienza entusiastica. Nelle manifestazioni tenute nei due giorni del suo soggiorno ebbero una parte di rilievo vari intellettuali maltesi, fra i quali gli scrittori in lingua italiana Ramiro Barbaro di San Giorgio e Zaccaria Roncali. Nello stesso anno il Barbaro pubblicò una relazione piena di ardore

109. E. Michel, L'isola di Malta focolaio di reazione legittimista 1860-1863, «A. S. M. », VII, fasc. III, 1936, pp. 304-333. 
a proposito della visita. ${ }^{110}$ Ruggiero Sciortino, consigliere di governo, fece un discorso al quale Garibaldi rispose cosi: " I maltesi dovrebbero molto amarmi, perchè molto li ho sempre amati, per la loro ospitalità a pro dei fratelli italiani e per il loro eroismo storico." Il popolo accorse numeroso e il generale salutò la grande folla che lo applaudì al grido di "Viva Garibaldi! Viva l'Italia!" Agenti borbonici, fra cui ex-poliziotti siciliani, tentarono, senza successo, di organizzare una controdimostrazione.

Ramiro Barbaro di San Giorgio dettò un indirizzo e lo fece sottoscrivere a centinaia di maltesi. Garibaldi rispose con le seguenti parole: "Mando una parola d'addio e di riconoscenza alla brava popolazione maltese, e l'accerto che giammai nella mia vita oblierò la fraterna accoglienza di cui volle onorarmi." Innumerevoli maltesi distinti visitarono il Generale, e furono presentati a lui da Nicola Fabrizi e dal ribelle maltese Emilio Sceberras, amico del Mazzini. Un'ultima grande acclamazione gli fu data quando lasciò l'isola a bordo del "Ripon", il 24 marzo."'

\section{I tardi esuli}

Caduta la monarchia borbonica in seguito alla spedizione dei Mille, sudditi siciliani e napoletani, nobili legittimisti e altri abbandonarono il loro paese ed emigrarono negli stati piu vicini. Malta ne attirò una grande parte. Nel primo periodo, dopo la presa di Palermo da parte delle falangi garibaldine, vi giunsero molti legittimisti che appartenevano alla nobiltà siciliana. Ma quando accadde il crollo finale, quasi tutti passarono nella capitale pontificia con l'intento di partecipare in qualche modo ad una restaurazione borbonica. Dopo il 1860 , gli emigrati siciliani a Malta si riducevano a pochi, e nell'estate $1864 \mathrm{i}$ legittimisti erano circa venti. ${ }^{112}$

Fra gli esuli che cercavano rifugio dopo la formazione del Regno d'Italia, c'era Gaetano Corleo, scrittore e poeta. Nel 1865 gli fu affidato l'incarico d'insegnare lingua e letteratura italiana nel "Convitto Ginnasio S. Giuseppe" in Valletta; e nel 1868 gli fu affidata la cattedra di lingua e letteratura all'università fino al 1905. Scrisse odi e canzoni, fra le quali una In morte del prof. G. A. Vassallo, il poeta maltese. ${ }^{113}$

Un caso molto interessante a proposito dei rapporti fra esuli e maltesi, e anche tra

110. R. Barbaro di San Giorgio, Garibaldi in Malta, Catania, A. Pastore, 1864

111. V. Laurenza. Garibaldi a Malta, estratto dall “A.S.M.”, III fasc. I-IV. pp.3-21

112. E. Michel, Emigrati barbonici a Malta 1864-1866, "A.S.M" II, fasc. IV 1931, pp. 210212.

113. L. Schiavone, op. cit., pp. 131-132 
letteratura in italiano e letteratura in maltese in questo periodo, è quello di Giuseppe Folliero de Luna, poeta, scrittore e musicista, che fece molte volte apparizione nell'isola prima di decidere di prendere stabile dimora, e infatti vi morì nel 1894. Legittimista e sostenitore del potere temporale del Papa, ${ }^{114}$ verso il 1850 ricevette a Malta la carica di Vice Console e poi di Console Generale delle Due Sicilie, ma dopo la caduta borbonica partì in esilio. L'esilio è argomento di una sua opera poetica intitolata L'Italiade - canto di un esule napoletano alla patria sperante, mentre a Malta pubblicò varie opere. ${ }^{115} \mathrm{Ma}$ il lavoro più significativo è un romanzo che scrisse in lingua maltese, Elvira jew imhabba ta' tirann. Nell'introduzione al libro, fra altre cose, fa un'apologia per il linguaggio italianeggiante che adopera e dichiara di essere maltese, benchè fosse sempre considerato napoletano: "Ghalchem jena imuieled Malta, izda cont ghadni zghajr meta sifirt biex nghamel li studji tighi fl'estru u uiehed jista jissoponi illi f'pajis fejn hatt jeu quasi hatt ma jitchellem il-lsien malti hua uisk facili illi uiehed jispicca jinsieh. Però jena ma inseitix ghal kollox il lingua maltia u meta tliet snin ilu argiajt geit gio dina il gzira cont ghadni nista nitchellem tajjeb jeu hazin."116

"Pur essendo nato a Malta, ero ancora piccolo quando partii per conseguire i miei studi all'estero, e si può supporre che in un paese dove nessuno o quasi nessuno parla la lingua maltese è molto facile dimenticarla. Ma io non ho dimenticato completamente la lingua maltese e quando tre anni fa feci ritorno in questa isola mi trovai ancora in grado di parlarla in qualche modo."

\section{L'Italianità e la sua difesa}

All'inizio del termine d'ufficio del Governatore Sir Arthur Borton (1878-1884), il governo britannico decise di inviare a Malta Sir Penrose Julyan e Patrick Keenan, il primo per investigare gli stabilimenti civili, e l'altro per analizzare il sistema educativo. Penrose Julyan, accanto alla vita civile, studio` altresi la possibilità, suggeritagli dal Segretario di Stato, di promuovere l'uso della lingua inglese come la lingua ufficiale dell'isola, particolarmente nel Servizio Civile, nelle scuole di governo, nel Consiglio di Governo e nelle corti di giustizia. Gli pareva che fosse deplorevole che, durante il corso di circa 80 anni in cui Malta faceva parte dell'impero britannico, non si

114. Cfr. Del Potere temporale dei papi e dell'Italia rispetto al pontificato romano, discorso di G. Folliero De Luna da Napoli, Malta, G. Grech e Co., 1849.

115. La lista delle sue opere include Studi critici sul restauro dell'antico dipinto dell'abside della chiesa di S. Giovanni in Valletta, Malta, Tip. del Portafoglio Maltese, 1868; La Chisciottiade poemetto bernesco, Malta, F. G. Caruana, 1872; Brevi rime seguite da tre saggi di poesia francese, Malta, Gpe. Maistre, 1883.

116. Elvira jew imhabba ta' tirann, rakkont storiku originali, Malta, S. Formosa, s.d., p. 4. 
fosse fatto alcuno sforzo per diffondere la conoscenza dell'inglese. A suo parere, l'incoraggiamento costante che si era dato all'italiano si trasformò in mezzo con cui gli agitatori politici trovarono una facilità "a guadagnare adepti alla loro teoria che i maltesi, quantunque di razza e di temperamento differenti, sono più affini agli italiani, e dovrebbero desiderare di essere uniti all'Italia, piuttosto che alla Gran Brettagna. ${ }^{117}$ Patrick Keenan, pur riconoscendo la diffusione dell'italiano nell'isola, tanto nella cultura quanto nelle scuole, condannò tutto, in modo particolare il sistema educativo e, mentre concesse una minima importanza al maltese, mantenne fermo che tutti dovevano sforzarsi di imparare 1 'inglese, e che questo doveva essere adottato come la lingua principale del paese, specialmente nell'educazione. ${ }^{118}$

Il movimento politico maltese, diretto da uomini che credevano con tutte le loro forze nell'antichità della tradizione italiana, anzi che il popolo maltese fosse un popolo italiano, ${ }^{119}$ non potevano accettare le nuove misure suggerite; e l'agitazione continuò a complicarsi e ad assumere nuove dimensioni. In quel periodo si era formato il partito "Anti-riformista", diretto da Fortunato Mizzi, con l'intento di combattere le riforme progettate da Julyan e da Keenan. Il concetto dell'italianità dell'isola fu propagato più che mai prima quando apparve nella scena politica il Mizzi, noto come "il padre della patria", cioè nel 1880 , quando fu nominato tra i rappresentanti del popolo nel Consiglio di Governo. L'agitazione politica cresceva anche in questa direzione linguistica; nella commemorazione della vittoria storica dei maltesi contro i turchi (1565), tenuta l'8 settembre 1882, un oratore gridava in piazza San Giorgio, a Valletta: "Malta è dei maltesi - fuori lo straniero!"'20 Fu il grido, tante volte ripetuto dai ribelli della penisola, che era destinato a dirigere i politici maltesi, soprattutto quelli che credevano con fervore nell'italianità tradizionale e nel pericolo futuro di rompere la continuità storica.

Quando i membri eletti, in seguito a controversie, elezioni, dimissioni e rielezioni, non approvarono il voto dell'educazione, perchè domandarono il sistema del "pari passu" per le due lingue - mentre il governo rifiutò di rinunziare al principio della libera scelta della lingua nei primi anni di scuola - il governo britannico ritirò la costituzione e poi la sostituì con quella del 1903. Come misura di protesta, i membri eletti ricorrevano ripetutamente alla politica dell'astensionismo. Nel 1910, ad eserfpio,

117. P. G. Julyan, op. cit., para. 308, p.. 56; Cfr. anche para. 311, p. 57.

118. P. J. Keenan, op. cit., para. XXIV-XL, pp. $91-94$ e passim.

119. Cfr. In difesa della civiltà italiana a Malta - raccolta di articoli di fondo del « Malta », organo del partito Nazionale Maltese a cura di A. Scicluna Sorge, Livorno, Giusti, 1931 passim.

120. E. Rossi, op.cit., p. 71. 
occorreva fare cinque elezioni generali. ${ }^{121}$ Le misure del governo inglese dirette alla diminuzione dell'importanza dell'italiano, specialmente nelle corti e nelle scuole, e all'introduzione dell' inglese, suscitarono nuove controversie. I giomali di quei decenni sono saturi di confessioni ardenti di italianismo e furono anche scritti vari libri da maltesi e da italiani, tutti partendo dalla premessa storica. ${ }^{122}$ La questione creo' un contraccolpo anche sui giornali e sulle riviste italiane che trattarono estensivamente del problema.

Il 19 dicembre 1901 un gruppo di intellettuali, politici e scrittori maltesi, tutti difensori della cultura italiana nell'isola, fondarono una nuova lega, la "Giovine Malta", un'imitazione, tanto nel nome quanto negli ideali che promisero di abbracciare, della società mazziniana. ${ }^{123}$ La maggior parte dei membri erano mizziani. ${ }^{124}$

L'entusiasmo che si associa alla vita degli esuli italiani nell'immediato passato, risale alla superficie nella descrizione che "La gazzetta di Malta" presentò della radunanza inaugurale:

Arturo Mercieca ${ }^{125}$ lesse un bellissimo discorso (...). Questo discorso fu interrotto

121. H. Ganado, op.,cit., p. 195

122. Cfr. A. Cini, La libera scelta ossia la questione della lingua in Malta secondo il Decreto Legge del 26 settembre 1901, Siracusa, Tip. del Tamburo, 1901; La grande mistificazione del Sig. Chamberlain ossia il valore delle concessioni fatte dall'Inghilterra a Malta ed all'Italia nella questione della lingua, Malta, 1902; La riconciliazione sulla questione della lingua ossia un disegno di riforma degli studi ginnasiali, Malta, Tip. del "Malta", 1903; Origine e progresso della lingua italiana in Malta ossia la lingua nazionale dei maltesi, Catania, G. Russo, 1903 (2a ed. 1931); B. Cellini, Malta e la politica stricklandiana, Livorno, Giusti, 1931; P. Fedele - R. Valentini, Per la storia dell'italianità di Malta nel Medio Evo, Roma, Sede dell'Istituto Palazzo dei Filippini, 1940; E. M. Gray, Le terre nostre ritornano ,- Malta-Corsica-Nizza, Novara, Istituto Geografico De Agostini, 1940; A. Scicluna Sorge, Malta: visione storica, sintesi politica, Livorno, Giusti, 1932; T. Andreucci, La lingua italiana e la cultura a Malta, Napoli, Hermes, 1935; C. Mallia, L'ultima fase della questione linguistica in Malta, Malta, Chretien \& Co., s.d.; E. Zammit, La questione della lingua a Malta a cospetto degli italiani, Siracusa, 1900.

123. Mazzini fondò il suo gruppo rivoluzionario dopo la prigionia, nel 1830 , con l'ideale di unire Ie forze per la costruzione di una repubblica italiana.

124. H. Ganado, op.cit., p.205

125. Il Mercieca (1878-1969), co-fondatore della “Giovine Malta", collaborava a diverse riviste letterarie e culturali, e fu attivissimo in campo politico. Assiduo sostenitore della cultura italiana a Malta, il Mercieca ha lasciato varie opera. Le più importanti delle quail sono Canti dell'esilio (1946) e Le mie Vicende (1947) 
frequentissimamente, quasi ad ogni proposizione, da fragorosi e prolungati applausi, da grida di bravo, e da promesse di cooperazione per raggiungere il descritto ideale. Pregato, parlò il presidente Dott. Fortunato Mizzi. Parlò quindi il sig. Giuseppe Howard ${ }^{126}$ e disse che nessun popolo si riscuoterebbe dalla schiavitù senza sacrifici, e che quando il paese avesse bisogno di vittime, egli sarebbe pronto a sacrificare la sua vita per la patria. Ragionava a lungo dello stato del paese e della necessità di rompere le catene della schiavitù.

Lo spirito risorgimentale italiano, intrecciato alla disposizione poetica del Mercieca, un letterato romantico, si fa vedere sotto vari aspetti: la visione dei moti politici e culturali maltesi come un "nostro risorgimento"; la ribellione contro la tirannia del dominio coloniale e l'ansia per la "santa" liberazione della patria; l'intimo legame tra tradizione culturale, particolarmente letteraria, e movimento di emancipazione politica; la fiducia nei giovani liberali; la consapevolezza dell'attività dei patrioti all'estero e il desiderio che il movimento locale vada avanti sulle loro orme; il riconoscimento della necessità di trovare il duplice metodo, di ascendenza mazziniana, di istruzione popolare e di pianificazione matura; la concezione della patria come il colmo di ogni idealità. Tutti questi motivi continuavano ad essere espressi con forza emotiva e declamatoria nella poesia italiana di vari poeti maltesi - Lorenzo De Caro (1817-1853), Paolo Cesareo (1844-1928), Antonio Dalli (1864-1948), Salvatore Castaldi (1856-1904), Giuseppe Mizzi (1873-1937), Luigi Arnaldo Randon (18761928), Vincenzo Frendo Azzopardi (18951955), Carmelo Mifsud Bonnici (1897-1948), Filippo Nicolò Buttigieg (1881-1969) - che si tuffarono nel mare della lotta nazionale, e non separarono il compito artistico dalla sfida politica, anzi tradussero la parola in un'arma; e invece della distillazione di sentimenti personali, cercavano di trarre fuori dallo spirito 1'invito alla partecipazione patriottica.

Le manifestazioni di adesione all'italianita`non mancarono. Ad esempio, nel 1905 una comitiva di studenti maltesi si reco' a Catania ed a Messina. In quell'occasione il Pascoli compose l'ode latina Ad sodales Melitenses che fu consegnata agli studenti; più tardi il poeta inviò la traduzione italiana e, accanto alla poesia, scrisse di suo pugno: "L'Italia vuol mantenere i suoi diritti; vuol conservare magari accrescere le sue conquiste ideali. Il pensiero e la sua spada l'idioma il suo scudo" Nel 1901,una comitiva di 700 maltesi si recò a Noto, in Sicilia, e fu accolta cordialmente; il capo del gruppo dichiarò in un suo discorso: "Siamo italiani, e italiani vogliamo rimanere a costo di perdere tutto, anche la vita. Il diritto e la ragione ci assistono (..). Siamo

126. Howard diventò più tardi primo ministro dell' isola (1921-1923). 
italiani. Ce lo dice la Religione che professiamo e soprattutto ce lo dice la lingua che parliamo e che vorrebbero togliere dalle nostre labbra." 127

\section{L'Intreccio delle lingue e l'accordo}

Nel 1905 si costitui I' “Associazione politica maltese”, diretta da Ignazio Panzavecchia, che poi nel 1910 fu chiamata "Comitato patriottico maltese"; ;28 sempre con lo scopo di ottenere per il popolo una costituzione che conceda maggiori poteri ai membri eletti.

L'acquisto dell'autonomia costituzionale era la prima vittoria importante dei maltesi nella loro marcia verso l'indipendenza, conseguita nel 1964. Dal 1921 in poi la nazione continuò a cercare la propria fisionomia, organizzando meglio il sistema dei partiti e superando la polemica linguistica nel 1934 allorchè il maltese, insieme con l'inglese, divenne lingua ufficiale. Attraverso gli assidui contatti con gli esuli italiani, col considerare le condizioni del risorgimento come analoghe alla situazione locale, il popolo maltese trovò l'ispirazione e la motivazione che gli mancavano. Alla base di tutto questo c'era il patrimonio culturale comune. ${ }^{129}$

Per interi secoli Malta svolgeva una vasta letteratura in italiano, il frutto di intelletti educati 'italianamente' che seguivano costantemente l'architettura stilistica e la gamma tematica degli autori della penisola. Quando poi ebbe inizio lo sviluppo di una letteratura in maltese, raggiungibile da tutti, benchè per lungo tempo priva di alte pretese artistiche, lo scrittore era in grado di interpretare fedelmente il sentimento proprio e collettivo, e non più l'ambizione accademica. L'autore non poteva più rinchiudersi nel santuario delle sue precettistiche e dei suoi formalismi, ma doveva incontrarsi con il popolo e ispirarsi alle sue esperienze. A Malta, il principio della popolarità della letteratura, un'eredità illuministica che il romanticismo modificò secondo le nuove profonde esigenze, non poteva realizzarsi pienamente in italiano.

127. E. Rossi, op. cit., pp. 80-81; O. F. Tencajoli, Poeti maltesi d'oggi, Roma, Angelo Signorelli, 1932, pp. 122 e 211-212.

128. H. Ganado, op. cit., p. 196.

129. Uno dei più antichi documenti italiani a Malta è del 1409 (cfr. Archivio della Cattedrale, Ms. A, ff. 171-176, pubblicato da A. Mifsud, Malta al sovrano nel 1409, "D", Ir, vol VIII, 7/1/1918, pp. 243-248) Cfr, anche A. Mifsud, La Cattedrale e l'Università, ossia il Comune e la chiesa in Malta. "D" II, vol II, 7/7/1917, pp. 39-40; U. Biscottini, Il volgare a Malta ed una questione dantesca. "G.P.L", X, vol. VI, 1934, pp. 665-670. 
Un tale processo di sviluppo in lingua maltese è nato circa nella prima metà dell'Ottocento - se si vuole parlare in termini di un movimento diffuso e piuttosto nazionale - quando chi scrisse in maltese non poteva prescindere dal fatto che, nonostante il sostrato semitico del suo idioma, la tradizione, la struttura dell'espressione e l'intera educazione letteraria di tutti (letterati e pubblico) erano esclusivamente italiane. Perciò la nuova produzione in maltese era inevitabilmente costretta a seguire la stessa direzione, e in effetti a mantenere la continuità storica che è sempre essenziale nell'evolversi del pensiero e della forma esterna che adotta. I1 detto sfondo è il quadro che, a cagione dell'inscindibile rapporto tra storia politica e attività creativa che la dottrina romantica tanto accentuava (particolarmente in Italia dove i problemi dell'identità e dell'unificazione nazionale furono sentiti più che altrove in Europa), spiega e giustifica le conquiste e le limitazioni del movimento romantico maltese. In tal modo la letteratura maltese, superando gradualmente gli svantaggi storici del pregiudizio e della noncuranza, poteva godere il vantaggio concesso dalla mentalità letteraria dell'epoca: il concetto del popolo, come il poeta che sente e scrive autenticamente, cioè di necessità, nella propria lingua. Mentre, da un canto, si continuò a scrivere la letteratura italiana della classe socialmente elevata, dall'altro lato le prime opere letterarie in maltese, benchè spesso scarse di alto valore estetico, toccavano l'animo del popolo e offrivano una sfida a chi provava la tentazione di sperimentare con una lingua 'incolta'.

Durante l'Ottocento e la prima metà del Novecento, lo scrittore in lingua maltese doveva far fronte alla duplice sfida di esprimersi (un problema comune ad ogni atto di estrinsecazione artistica) e di elevare con dignità un veicolo non curato al livello di lingua letteraria (una caratteristica della condizione storica). ${ }^{130}$ Ciò significa che, in ultima analisi, la polemica tra l'italiano (come la lingua rispettata della classe colta) e il maltese (come la lingua parlata piú antica dell'isola e l'idioma più vicino alle

130. La costituzione del 1921, per mitigare le controversie, non diede alcun riconoscimento alla lingua maltese e riteneva un grado di importanza uguale all'italiano e all'inglese (cfr. Sezione 57). Fu soltanto nel 1934 che il maltese, diventato finalmente lingua ufficiale dell'isola, fu ammesso nel curricolo delle scuole governative, nelle corti di giustizia e negli atti legislativi per mezzo di emendamenti costituzionali. Nello stesso anno fu riconosciuto ufficialmente il sistema ortografico dell' "Ghaqda tal-Kittieba tal-Malti", fondata nel 1920 (cfr. N. Cremona, Antologija ta' proża Maltija, Malta, Malta University Press, 1970, p. 32). 
masse), ${ }^{131}$ pur dividendo e alienando il popolo per lungo tempo, ${ }^{132}$ non doveva influire gravemente sul procedimento del maltese come mezzo letterario e sulle vie da essere battute dalla nuova letteratura in sede tematica e stilistica. Si doveva trattare di una sintesi tra la tradizione e il presente, non di una completa rottura. Gli autori maltesi, infatti, ritenevano il ricco deposito che avevano ereditato da varie fonti (tradizione nazionale, educazione, consapevolezza della vicinanza di Malta alla penisola), e lo modificarono quasi istintivamente in maltese. Alla base di questa loro disposizione a non distinguere tra la cultura ereditata e la cultura che si aspettava da loro, c'è l'incapacità di distinguere nettamente tra cultura italiana e cultura maltese, anzi l'inclinazione a vedere nell'una il prototipo dell'altra.

Una conclusione diversa dovrebbe spiegare 1) perchè la continuità storica della letteratura a Malta (prima in italiano e poi in maltese) non è rotta; 2) quale contenuto umano, se non quello della spiritualità latina a cui l'isola partecipava con naturalezza, poteva essere sentito ed espresso da un autore locale. Una posizione contraria a queste considerazioni suggerisce che l'autore maltese poteva ignorare il mondo dell'immediatezza, le condizioni di spazio di tempo (indispensabili come dati aprioristici per ogni creazione artistica, specialmente romantica) e produrre in un vuoto. Il movimento romantico maltese (quasi coestensivo con tutto il periodo dell'esistenza di una letteratura scritta in quella lingua, in verità dall'inizio fino agli anni sessanta del Novecento, quando si ebbe una severa polemica contro la tradizione da una nuova generazione di autori) riconosce le radici della cultura dell'isola e utilizza l'eredità straniera come materia di costruzione per la rielaborazione di una esperienza che, benchè autentica, si rifà mediante le forme che esistono e che non vanno oltre $i$ confini in cui si dibatte il mondo italiano.

È importante, ad esempio, ricordare che la lingua maltese, pur avendo una sottostruttura semitica, è scritta nell'alfabeto latino; ${ }^{133}$ la sua poesia tradizionale non si costruisce secondo le regole prosodiche di qualche dialetto di origine semitica, o secondo la struttura del canto ebraico o di quello che si compone nel Medio Oriente,

131. Gli arabi occuparono l'isola nel 870 . La tribù di 'Aghlabid' che aveva conquistato il paese rimase a Malta per 220 anni. Durante tale periodo riuscì a imporre la sua lingua sulla piccola comunita` nativa (cfr. G. Aquilina, Papers in Maltese, linguistics, Malta, Royal University of Malta, 1961,pp. 45-46).

132. Cercando una linea generale di demarcazione, si può collocare la questione della lingua, come problema che assume dimensioni nazionali, nel periodo 1880-1939 (cfr. H. Frendo, Language and nationality in an island colony: Malta, «C.R.S.N.», I, vol. III, 1975, p. 25).

133. G. Aquilina analizza i vari sistemi ortografici proposti dai primi scritti di G. F. Bonamico 
ma secondo la metrica italiana. ${ }^{134}$ Ma più sostanziale di queste qualita` elementari è ancora l'unità di spiriti e di tendenze che distingue letteratura maltese da quella di altre nazioni che hanno anch'esse una lingua di base semitica. Si tratta di un risultato complesso di una tradizione che si formò ininterrottamente durante vari secoli; e di un ripensamento storico - che coincide con il rinnovamento artistico, patriottico e sociale del romanticismo storico - che era possibile perchè l'isola si trovava così vicina alla storia e alla spiritualità dell' Italia.

(1672-1675) fino al 1934 (quando il sistema dell' “Ghaqda tal-Kittieba tal-Malti” raggiunse il livello scientifico); e distingue nettamente i romanisti e gli arabisti: "The Arabists were those who, basing themselves on the fact that Maltese is a semitic language, akin to Arabic or, as some others wrongly believed, to Hebrew, favoured the use of the Arabic alphabet. Thank heavens, the Arabists did not have their way for the system would have certainly cut us off from the literature of the countries where the Latin script is used" (Papers in Maltese linguistics cit., p. 81). "Gli Arabisti erano quelli che, basandosi sui fatto che il maltese è di origine semitica, affine all' arabo o, come altri hanno creduto erroneamente, all'ebraico, favorivano l'uso di un alfabeto arabo. Grazie al cielo, gli Arabisti non riuscirono a introdurlo, altrimenti quel sistema certamente ci avrebbe separato dalla letteratura di quei paesi che adoperano l'alfabeto latino."

134. Cfr. K. Vassallo, Metrika Maltija, Malta, A. C. Aquilina \& Co., 1942; e Vatum consortium, Malta, Dar ta' S. Guzepp, 1968. 